Draft VERsion July 1, 2018

Preprint typeset using $\mathrm{LATE}_{\mathrm{E}} \mathrm{X}$ style emulateapj v. 5/2/11

\title{
A COLD FLARE WITH DELAYED HEATING
}

\author{
Gregory D. Fleishman ${ }^{1,2}$, Valentin D. Pal'shin ${ }^{2}$, Natalia Meshalkina ${ }^{3}$, Alexandra L. Lysenko ${ }^{2}$, Larisa K. \\ Kashapova $^{3}$, Alexander T. Altyntsev ${ }^{3}$ \\ Draft version July 1, 2018
}

\begin{abstract}
Recently, a number of peculiar flares have been reported, which demonstrate significant nonthermal particle signatures with a low, if any, thermal emission, that implies close association of the observed emission with the primary energy release/electron acceleration region. This paper presents a flare that appears a "cold" one at the impulsive phase, while displaying a delayed heating later on. Using HXR data from Konus-Wind, microwave observations by SSRT, RSTN, NoRH and NoRP, context observations, and 3D modeling, we study the energy release, particle acceleration and transport, and the relationships between the nonthermal and thermal signatures. The flaring process is found to involve interaction between a small and a big loop and the accelerated particles divided in roughly equal numbers between them. Precipitation of the electrons from the small loop produced only weak thermal response because the loop volume was small, while the electrons trapped in the big loop lost most of their energy in the coronal part of the loop, which resulted in the coronal plasma heating but no or only weak chromospheric evaporation, and thus unusually weak soft X-ray emission. Energy losses of fast electrons in the big tenuous loop were slow resulting in the observed delay of the plasma heating. We determined that the impulsively accelerated electron population had a beamed angular distribution in the direction of electric force along the magnetic field of the small loop. The accelerated particle transport in big loop was primarily mediated by turbulent waves like in the other reported cold flares.

Subject headings: acceleration of particles - diffusion - magnetic fields — Sun: flares - Sun: radio radiation-turbulence
\end{abstract}

\section{INTRODUCTION}

A close casual relationship between the nonthermal particles accelerated in flares due to release of the excessive magnetic energy and plasma heating has come to be known as the Neupert effect (Neupert 1968). Specifically, Neupert (1968) discovered that the soft $\mathrm{X}$-ray (SXR) light curves in a number of flares at the rise phase and up to the SXR peak were well correlated with the running time integral of the impulsive microwave emission from the flare. Currently, the Neupert effect is commonly referred to a similar relationship between the impulsive hard X-ray (HXR) and thermal SXR emissions. One way or the other, the Neupert effect suggests that (at least in some flares) the particle acceleration takes place first giving rise to nonthermal microwave and HXR emissions and then the energy and momentum losses of these accelerated particles result in the thermal response in the form of coronal plasma heating and/or chromospheric evaporations; the heated coronal plasma is then cooling down relatively slowly due to conductive and radiative losses.

It was long ago established (e.g., Dennis 1988) that the impulsive flares demonstrating a clear Neupert effect represent only a fraction of all events. Most recently, with the spectrally resolved X-ray data obtained with Reuven Ramaty High Energy So-

\footnotetext{
${ }^{1}$ Center For Solar-Terrestrial Research, New Jersey Institute of Technology, Newark, NJ 07102

${ }^{2}$ Ioffe Institute, St. Petersburg 194021, Russia

3 ISZF, Irkutsk, Russia
}

lar Spectroscopic Imager (RHESSI, Lin et al. 2002) many more low-energy gradual (presumably, mostly thermal) events have been detected. For example, Su et al. (2008) find that about $2 / 3$ of all events are gradual, up to $10 \%$ are impulsive, and up to $20 \%$ are early impulsive flares (Sui et al. 2006, 2007); only a fraction of the latter class events demonstrates a clear Nuepert effect, which suggests that the relationships between nonthermal and thermal energies are much more complex in the general case (e.g., Veronig et al. 2005) than the simple loss-to-heating correspondence implied by the standard Neupert effect.

It has recently been recognized that some early impulsive flares are in fact 'cold flares' (Bastian et al. 2007; Fleishman et al. 2011; Masuda et al. 2013) in which no or so modest thermal plasma response is detected that these events are not listed as GOES flares. The three reported cold flares, although all similar in a lack of the thermal response, are, however, noticeably different from each other in a number of other respects. For example, the 30-Jul-2002 cold flare reported by Fleishman et al. (2011) is a 'tenuous' flare with the thermal number density not exceeding $2 \times 10^{9} \mathrm{~cm}^{-3}$ at the coronal part of the flaring loop. In such cases the plasma heating due to fast electron collisions with the coronal thermal particles is small because the collisions are rare in the tenuous plasma, while the chromospheric evaporation is suppressed by some reason. On the contrary, two other cold flares reported by Bastian et al. (2007) and Masuda et al. (2013) were dense with the thermal number density in excess of $10^{11} \mathrm{~cm}^{-3}$. In such 


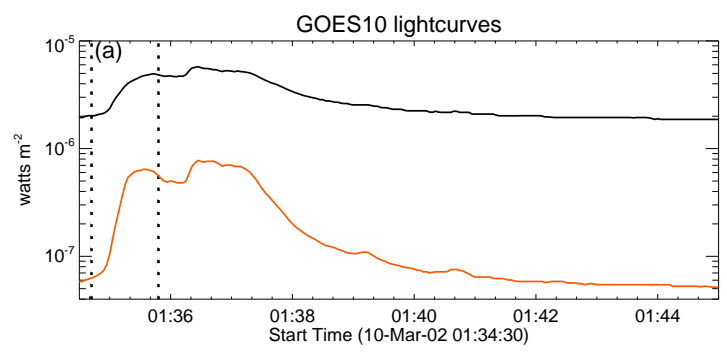

Synthetic (NoRH+RSTN) dynamic spectrum
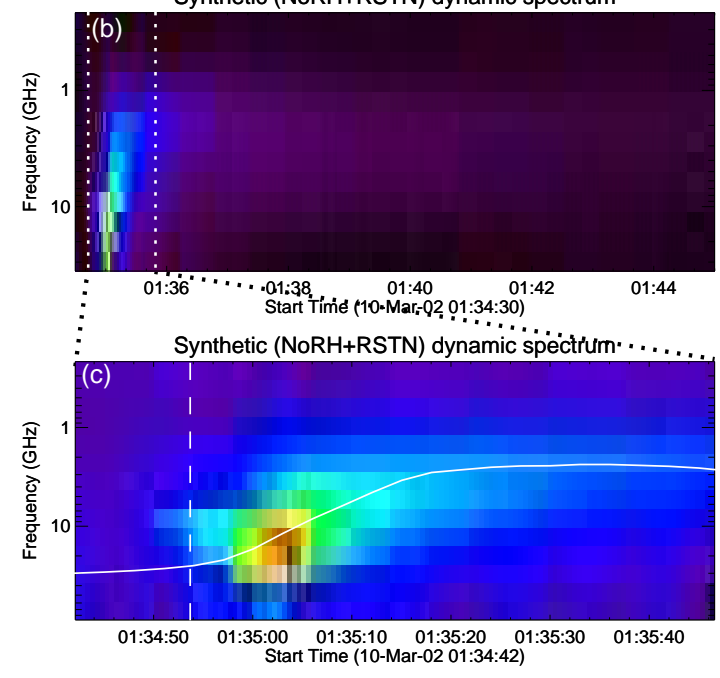

$\mathrm{KW}$ lightcurves

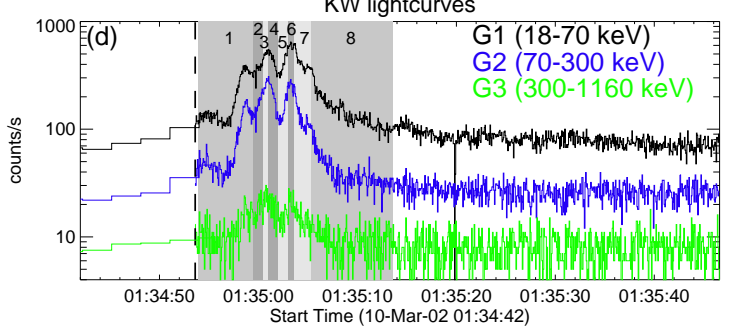

FIG. 1.- Overview of March 10, 2002 flare. (a) GOES (3 s) lightcurves as measured by GOES-10 spacecraft. (b) microwave dynamic spectrum. (c) zoom-in dynamic spectrum of the impulsive flare phase. White solid curve shows the highenergy GOES lightcurve. (d) Konus- Wind lightcurves in three energy bands. Dotted vertical lines in $(\mathrm{a}, \mathrm{b})$ demarcate the impulsive phase shown in panels (c) and (d). Dashed vertical lines in (c,d) show the start time of Konus- Wind fast record. The vertical stripes shown by dark or light grey background denote the eight intervals over which we extracted the spectra in Figure 3

cases the fast particle losses in the coronal part of the loop are large and the increase of the thermal energy is relatively strong; but, because of high density, the net temperature increase above the coronal preflare level is rather modest.

The Neupert effect is clearly present in the reported cold flares in its nominal form, i.e., the time derivative of the SXR light curve closely correlates with the light curves of the nonthermal HXR and microwave emissions. Morphologically, the microwave emission in all reported cold flares has a coronal (loop-like) structure, while X-ray morphology differs depending on if the flare is dense or tenuous: the dense flares demonstrate a coronal X-ray source, while the tenuous flare - two chromospheric foot points; the coronal part of the cold flare sources has been identified with the very acceleration region of fast electrons (Fleishman et al. 2011). Timing of these events is inconsistent with purely collisional transport of fast electrons, while requires the transport mediated by wave turbulence (Bastian et al. 2007; Fleishman et al. 2011). The spectra of fast electrons are typically hard in the cold flares, with the spectral index $\delta \sim 3.5$. Overall, the cold flares appear to be events with efficient electron acceleration but only a modest plasma heating. It appears that the heating is entirely supplied by energy losses of the accelerated electrons without any apparent additional heating. Therefore, compared with other flare types, the nonthermal-energy-dominated cold flares offer a cleaner way of studying electron acceleration in flares and their effect on subsequent plasma heating. Thus, both the acceleration of electrons and the nonthermal-to-thermal energy evolution can be studied much more conclusively in the cold flares then in a 'normal' flare.

This paper presents an unusual case of a solar flare, which shows only a very mild thermal emission during the entire impulsive phase like other cold flares, but then demonstrates a more substantial heating that lasts considerably longer than the impulsive phase with the heating rate comparable to that observed at the impulsive phase. We argue that this behavior can be understood if two interacting loops are involved in the flare - a small one and a large one. The small loop, presumably the region of its interaction with the big loop, plays a role of particle accelerator and particle injector for the larger loop, where the fast electrons are effectively accumulated. During the impulsive phase the flare thermal response is driven by the fast electron losses from the small loop, but laterby the same losses of the trapped population from the large loop. From the X-ray and microwave data augmented by 3D modeling, we estimate the key source parameters, such as the total number of the nonthermal electrons, the spectral shape, and even the pitchangle distributions at a few time frames, and discuss the corresponding implications for the particle acceleration, plasma heating, and thermal-to-nonthermal energy partitions.

\section{OBSERVATIONS}

\subsection{Data set overview}

The solar flare, GOES class C5.1, occurred on 10 March 2002 nearby the eastern solar limb in AR 09866; see summary of the total power data in Figure 1. HXR and gamma-ray data for this event are obtained with the Konus- Wind (KW) spectrometer (Aptekar et al. 1995), while the Reuen Ramaty High Energy Solar Spectroscopic Imager (RHESSI, Lin et al. 2002) data were not available because of the RHESSI night.

Konus-Wind is a joint US-Russian experiment launched on November, 1, 1994 to study the gammaray bursts and solar flares. It consists of two $\mathrm{NaI}(\mathrm{Tl})$ detectors S1 and S2 observing correspondingly the northern and southern celestial hemispheres. Unlike RHESSI, this instrument operates in the interplanetary space (since 2004-near Lagrange point L1), 
so it does not suffer from "nights", and, thus, has a very high duty cycle of about $95 \%$. Thanks to being far from the Earth's magnetosphere it has an exceptionally stable background. Konus-Wind works in two modes: waiting mode and trigger mode. In the waiting mode the count rate light curves are available in three wide energy channels $\mathrm{G} 1(\sim 18-70 \mathrm{keV})$, $\mathrm{G} 2(\sim 70-300 \mathrm{keV}), \mathrm{G} 3(\sim 300-1160 \mathrm{keV})$ with accumulation time $2.944 \mathrm{~s}$. In the trigger mode KonusWind measures count rate light curves in the same three channels with a varying time resolution from 2 to $256 \mathrm{~ms}$ and with total duration of $250 \mathrm{~s}$. While in the trigger mode, 64 multichannel spectra are taken in addition to the light curves as follows. The multichannel spectra are measured in two partially overlapping energy bands: $\sim 20-1150 \mathrm{keV}$ and $\sim 240 \mathrm{keV}-$ $15 \mathrm{MeV}$ in 2002 year. Each band has 63 energy channels with fixed nominal boundaries. Accumulation time of the first four spectra is fixed at $64 \mathrm{~ms}$ and of the last eight spectra-at $8.192 \mathrm{~s}$. For the remaining 52 spectra the accumulation time is adaptively adjusted from $0.256 \mathrm{~s}$ to $8.192 \mathrm{~s}$ based on the count rate in the $\mathrm{G} 2$ channel: for more intense events the accumulation time is proportionally shorter. Switch on to the trigger mode occurs at a statistically significant excess above a background count rate within an interval of $1 \mathrm{~s}$ or $140 \mathrm{~ms}$ in the G2 energy channel (Pal'shin et al. 2014).

The flare triggered the Konus- Wind detector S2 at $t_{0}(\mathrm{KW})=5693.874 \mathrm{~s}$ UT $(01: 34: 53.874)$. The propagation delay from WIND to the center of the Earth is $0.241 \mathrm{~s}$ for this flart; time corresponds to the Earthcrossing time 01:34:54.115 UT.

Microwave total power (TP) data are obtained with the Nobeyama RadioPolarimeters (NoRP, Torii et al. 1979) in intensity and circular polarization at six frequencies $(1,2,3.75,9.4,17, \& 35 \mathrm{GHz})$ and in the intensity only at $80 \mathrm{GHz}$ with the time resolution $0.1 \mathrm{~s}$ during the impulsive peak, 01:34:36-01:35:46 UT, and $1 \mathrm{~s}$ outside the peak (accordingly, no $80 \mathrm{GHz}$ data), as well as $1 \mathrm{~s}$ intensity data from the Radio Solar Telescope Network (RSTN; Guidice et al. 1981) at seven frequencies $(0.4,0.6,1.4,2.7,5.0$, 8.8 , \& $15.4 \mathrm{GHz} 5$. The flux at $80 \mathrm{GHz}$ was adjusted using the time-dependent correction coefficient $k_{\text {corr }}=\left[T_{\text {years }} / 1995.83\right]^{630}$ (Nakajima (2007) private communication; see also Altyntsev et al. 2008; Kundu et al. 2009), while the polarization at $1 \& 2$ $\mathrm{GHz}$ were corrected for the differing gains in the $I$ and $V$ channels (Shibasaki (2007) private communication; see also Altyntsev et al. 2008). Using these heterogeneous sources of the microwave data we built two complementary dynamic spectra of the microwave burst. The first of them straightforwardly combines daily NoRP data with RSTN data, both of which have $1 \mathrm{~s}$ time (slow) resolution, to form a synthetic dynamic spectrum in the $0.4-35 \mathrm{GHz}$ range. This dynamic spectrum is, however, insufficient for our anal-

\footnotetext{
4 The corresponding delay to the Nobeyama Observatory whose microwave data are used for the analysis is $0.231 \mathrm{~s}$.

5 To correct for RSTN clock and amplitude calibration errors, the RSTN time was shifted as $t_{\text {true }}=t_{\text {obs }}-3.3 \mathrm{~s}$, the light curve at $8.8 \mathrm{GHz}$ was corrected by the factor of 1.4 and at $15.4 \mathrm{GHz}$ by the factor of 1.55 .
}

ysis for two reasons: during the impulsive peak the emission (1) shows subsecond variations and (2) has a high-frequency spectral peak around $35 \mathrm{GHz}$. Thus, the fast NoRP record made in the burst mode $(0.1 \mathrm{~s})$ containing the $80 \mathrm{GHz}$ data is essential for the analysi:6. We employ these high time resolution data in two ways. For the light curve and polarization data analysis we use the full time resolution of $0.1 \mathrm{~s}$. But for the spectral analysis we also created a 'fast' dynamic spectrum with $0.5 \mathrm{~s}$ resolution, which combines the NoRP $0.1 \mathrm{~s}$ data resampled to $0.5 \mathrm{~s}$ steps with interpolated (from $1 \mathrm{~s}$ to $0.5 \mathrm{~s}$ ) RSTN data. Adding the RSTN data, although compromises the time resolution, is important for the spectral analysis because the NoRP data alone have too few data points for a meaningful spectral fit.

The microwave imaging is performed with the Siberian Solar Radio Telescope (SSRT) at $5.7 \mathrm{GHz}$ (intensity and polarization) and the Nobeyama RadioHeliograph (NoRH, Nakajima et al. 1994) at 17 $\mathrm{GHz}$ (intensity and polarization) and $34 \mathrm{GHz}$ (intensity only). The SSRT is cross-shaped interferometer and the data recorded by the EW and NS arrays provide two-dimensional images of the solar disk every two-three minutes and one-dimensional images every $0.3 \mathrm{~s}$ in the standard mode of the observations (Grechnev et al. 2003). The methods of analysis for one-dimensional solar images have been described by Altyntsev et al. (2003) and Lesovoi and Kardapolova (2003). The receiver system of SSRT contains a spectrum analyzer with $120 \mathrm{MHz}$ frequency coverage using an acousto-optic detector with 250 frequency channels, which correspond to the knifeedge-shaped fan beams for the NS and EW arrays. The frequency channel bandwidth is $0.52 \mathrm{MHz}$. The response at each frequency corresponds to the emission from a narrow strip on the solar disk whose position and width depend on the observation time, array type, and frequency. The signals from all the channels are recorded simultaneously and generate a one-dimensional distribution of solar radio brightness. During the event under study the width of the beam of SSRT was $18^{\prime \prime}$ for EW array and $30^{\prime \prime}$ for NS array.

A limited information about the thermal plasma at the flare region is available from a few images taken at $195 \AA$ with Extreme ultraviolet Imaging Telescope (EIT) onboard Solar \& Heliospheric Observatory (SoHO/EIT, Domingo et al. 1995) with the 12 min cadence. The context SXR GOES-10 data and the line-of-sight magnetogram from the Michelson Doppler Imager (SoHO/MDI Domingo et al. 1995) are utilized. The line-of-sight magnetogram is used for the 3D modeling with the GX Simulator (Nita et al. 2015).

\subsection{Light curves}

A striking feature of this flare is a contrasting combination of the impulsive and gradual light curves is vividly illustrated in Figure 2, a prominent impulsive emission is apparent in the KW light curves and in

6 The original $80 \mathrm{GHz}$ data are very noisy; they were smoothed using $4 \mathrm{~s}$ window before inclusion into the dynamic spectrum. 

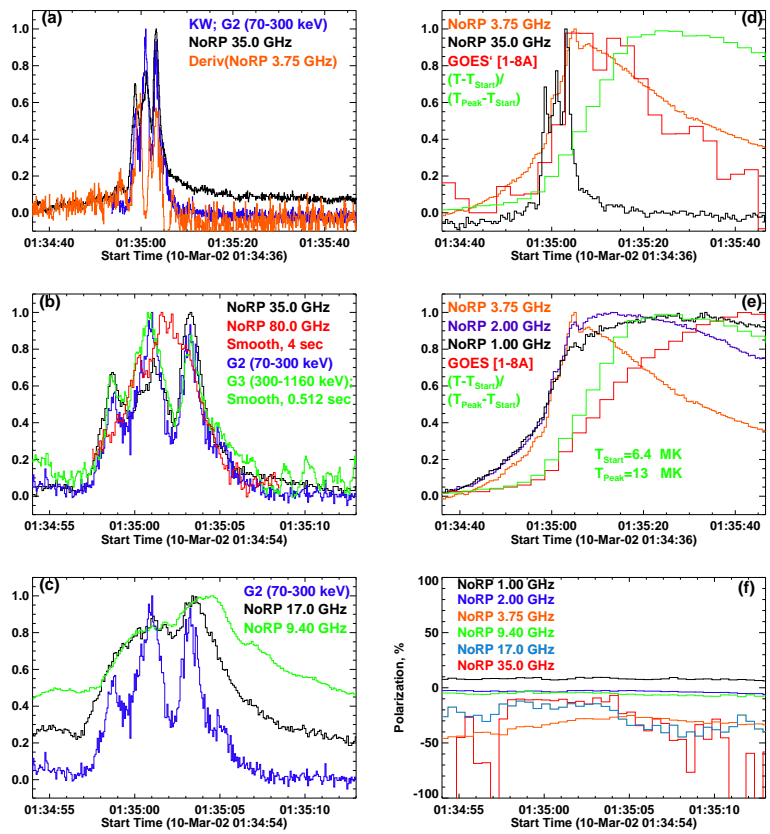

FIG. 2.- Summary of the normalized light curves. (a) Overall comparison of the impulsive microwave and HXR light curves at $35 \mathrm{GHz}(0.1 \mathrm{~s}$ cadence) and $70-300 \mathrm{keV}$ (64 ms cadence) with the time derivative of the gradual microwave light curve at $3.75 \mathrm{GHz}$. (b) Microwave light curves at $35 \& 80 \mathrm{GHz}$ along with Konus-Wind HXR G2 and G3 light curves. (c) Microwave light curves at $9.4 \& 17 \mathrm{GHz}$ in comparison with Konus- Wind HXR G2 light curve. (d) Microwave light curves at $3.75 \& 35 \mathrm{GHz}$ along with time derivative of the GOES(1$8 \AA$ ) light curve and GOES-derived plasma temperature evolution $\left(T_{\text {Start }} \approx 6.4 \mathrm{MK} ; T_{\text {Peak }} \approx 13 \mathrm{MK}\right)$. (e) Microwave light curves at 1,2 , \& $3.75 \mathrm{GHz}$ along with $\operatorname{GOES}(1-8 \AA)$ light curve and GOES-derived plasma temperature. (f) The degree of polarization of the microwave emission at the impulsive flare phase. Note the increase of the degree of polarization at $17 \&$ $35 \mathrm{GHz}$ at the decay phase.

the high-frequency microwave light curve at $35 \mathrm{GHz}$, while the microwave light curves are getting progressively more gradual towards the lower frequencies; the GOES light curves are even more gradual than the low-frequency microwave light curves. Thus, this section pays a close attention to these various light curves and relationships between them.

Figure 2a shows a general similarity between the microwave light curve at $35 \mathrm{GHz}$, the HXR KonusWind G2 light curve at 70-300 keV, and time derivative of the microwave light curve at $3.75 \mathrm{GHz}$. Although these three light curves differ from each other in some details, the overall high correlation between them is apparent; no delay is seen between the light curves.

Figure 2] compares the high energy Konus-Wind channels G2 and G3 with the high-frequency microwave $35 \mathrm{GHz}$ and $80 \mathrm{GHz}$ light curves over the impulsive phase of the burst. The $35 \mathrm{GHz}$ light curve is as closely correlated with each of the HXR light curves as the HXR light curves correlate with each other. Again, no delay between the $35 \mathrm{GHz}$ and HXR light curves is apparent within the instrumental time resolution ( $64 \mathrm{~ms}$ in case of Konus- Wind and $100 \mathrm{~ms}$ in case of NoRP). On the contrary, the $80 \mathrm{GHz}$ light curve does not correlate in detail with all other light curves, which is the result of the already mentioned 4-s smoothing of the $80 \mathrm{GHz}$ light curve needed to reduce the high fluctuation level in the original signal. Figure 2k displays a similar relationship, but between the Konus- Wind G2 light curve and the microwave light curves at $17 \mathrm{GHz}$ and $9.4 \mathrm{GHz}$. In this case, the microwave light curves are less impulsive than and delayed relative to the HXR light curve, while the shapes of the microwave light curves at 9.4 \& $17 \mathrm{GHz}$ are closely correlated with each other at the rise phase, where they appear earlier than the most impulsive light curves.

Figure $2 \mathrm{~d}$ compares, at the first place, the impulsive $35 \mathrm{GHz}$ light curve and the derivative of the $\operatorname{GOES}(1-8 \AA)$ light curve. In contrast to the expectation based on the standard Neupert effect, these two light curves do not correlate to each other: even though the GOES derivative does reach the peak value at the impulsive peak, it appears strongly delayed relative to the impulsive light curve. For further reference, this panel also displays the GOES-derived evolution of the plasma temperature and a more gradual microwave light curve at $3.75 \mathrm{GHz}$, which shows a much closer correlation with the GOES derivative. Then, Figure 20 displays all the gradual lowfrequency microwave light curves, the GOES $(1-8 \AA)$ light curve, and the temperature evolution. This comparison shows that the light curves are getting more and more gradual and delayed at lower frequencies, with the GOES light curve being the most delayed. This delay, even though appears small (about $40 \mathrm{~s}$ ) by the absolute value, is highly substantial indicating the heating process that is roughly four times longer than the duration of the impulsive phase. The temperature light curve is well correlated with the $1 \mathrm{GHz}$ light curve at their peak phase. It is interesting to recall here, Figure 2 a, that the impulsive $35 \mathrm{GHz}$ and HXR light curves are well correlated with the time derivative of the $3.75 \mathrm{GHz}$ light curve (rather than SXR light curve). This correlation indicates that the $3.75 \mathrm{GHz}$ light curve either represents the plasma thermal response on the accelerated electron impact or corresponds to a trapped population of fast electrons, whose injection profile corresponds to the $35 \mathrm{GHz}$ or Konus- Wind light curves.

Figure 2f shows evolution of the degree of polarization of the microwave emission during the impulsive peak. Two interesting features are to be noted about this figure: (i) an unexpectedly high degree of polarization at $3.75 \mathrm{GHz}$ during the entire event7, indicating an optically thin emission at these intermediate frequencies, and (ii) the degree of polarization at $17 \&$ $35 \mathrm{GHz}$ is getting larger at the decay of the impulsive phase, which may imply substantial modification of the angular distribution of the nonthermal electrons; we return to these points later.

\subsection{Spectra}

\subsubsection{X-ray Spectra}

We performed analysis of the Konus- Wind spectral data for eight time intervals indicated in Figure 11,

7 A comparably strong polarization (not shown in the figure) is detected at $5.7 \mathrm{GHz}$ with SSRT. 

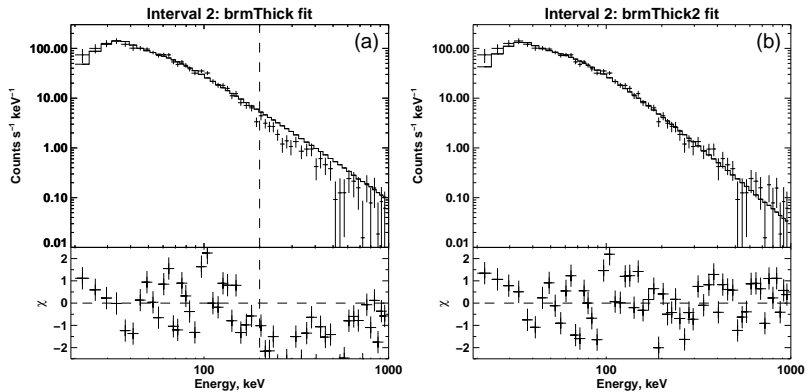

FIG. 3.- Konus- Wind spectrum of the flare for time interval \#2 (Fig. 1 d) with the fit examples: (a) brmThick model fitted to the photon energy range $20-200 \mathrm{keV}$ demarcated by the vertical dashed line, (b) brmThick2 model fitted to the full range of $20-1000 \mathrm{keV}$.

where the signal exceeded the background. For the analysis the energy channels were rebinned to contain at least 20 counts per energy bin in each time interval and fitted in various energy subranges within the 20$1000 \mathrm{keV}$ range, as detailed below. Despite the emission is seen up to $\sim 10 \mathrm{MeV}$, we did not include the channels above $1 \mathrm{MeV}$ since they can contain a significant contribution of nuclear line emission, which is not discussed here.

We fit the spectra with a number of alternative spectral models. Unambiguously, a single power-law (either of the electron or photon spectra) is inconsistent with the data, when the whole range of the photon energies, $20-1000 \mathrm{keV}$, is analyzed. On the other hand, when a broken power-law model is applied for either electron or photon spectrum, the fit parameters are returned with rather large uncertainties, which implies that a wide range of spectral models is consistent with the data. In particular, a single power-law can fit the data reasonably well, when a truncated photon energy range is selected, e.g., 20$200 \mathrm{keV}$ (Figure 3a), 40-400 keV, or 100-1000 keV, which may imply that the spectral steepening progresses slowly but steadily with energy. A number of instrumental or physical effects are known to yield spectral flattening at low energies, namely, photon pile-up, photospheric albedo, nonuniform ionization of the target, and return current. We checked via modeling that the pile-up plays no role in our case. Other mentioned effects typically play a role at low energies, $E \lesssim 50 \mathrm{keV}$ (Holman et al. 2011), while in our flare the spectral break happens at much higher energy $E \gtrsim 100 \mathrm{keV}$. Nevertheless, we employed the fit with albedo correction, but this did not improve the goodness of fit. Also we superimposed thermal bremsstrahlung model with temperature $\sim 14 \mathrm{MK}$ and emission measure $\sim 2 \times 10^{48} \mathrm{~cm}^{-3}$ as estimated from the GOES peak flux on our spectra and found that the thermal contribution does not exceed $1 \%$ even at the lowest energy channels. As a result of our tests we conclude that the nonthermal electron spectrum has a convex shape (the high-energy slope is steeper than the low-energy one). Out of the variety of the considered spectral models we present here some results for three models, using XSPEC 12.5 (Arnaud 1996).

The first of them is a phenomenological broken power-law model, $B P L$, taken in the form:

$$
I(E)= \begin{cases}A\left(\frac{E}{100 k e V}\right)^{-\gamma_{1}} & E \leq E_{b r, p h} \\ A E_{b r, p h}^{\gamma_{2}-\gamma_{1}}\left(\frac{E}{100 k e V}\right)^{-\gamma_{2}} & E_{b r, p h}<E\end{cases}
$$

where $\gamma_{1}$ and $\gamma_{2}$ are the $P L$ photon indexes and $A$ is the normalization at $100 \mathrm{keV}$ in units of photons $\mathrm{cm}^{-2} \mathrm{~s}^{-1} \mathrm{keV}^{-1}$.

The other two are the collisional thick-target models assuming a power-law (brmThick) in the fast electron flux spectrum (el's/keV/s) over energy between the low- and high- energy cutoffs:

$$
F(E)= \begin{cases}0 & E<E_{\text {cut }, \text { low }} \\ \propto E^{-\delta} & E_{\text {cut }, \text { low }} \leq E \leq E_{\text {cut }, \text { high }} \\ 0 & E_{\text {cut }, \text { high }}<E\end{cases}
$$

and a broken power-law electron spectrum (brmThick2):

$$
F(E)= \begin{cases}0 & E<E_{\text {cut }, \text { low }} \\ \propto E^{-\delta_{1}} & E_{\text {cut }, \text { low }} \leq E \leq E_{b r, e} \\ \propto E^{-\delta_{2}} & E_{b r, e} \leq E \leq E_{\text {cut }, \text { high }} \\ 0 & E>E_{\text {cut }, \text { high }},\end{cases}
$$

normalized to the total flux of the electrons [electrons/s] above $E_{\text {cut,low. }}$. Since the XSPEC package does not contain standard models of the thin or thick targets from a broken power-law distribution of the nonthermal electrons, routinely used for the analysis of X-ray spectra of solar flares, these models were added by us to XSPEC based on analogous models used in the OSPEX package (Schwartz et al. 2002). We also performed the corresponding fitting using the OSPEX package from SSW/IDL to cross-check the fitting results and found that the fitting parameters are fully consistent with each other.

Given that the GOES flux was somewhat low during the impulsive flare phase, we examined if the GOES data can constrain the low-energy cut-off in the accelerated electron spectrum. But in fact, no conclusive constraint was obtained, perhaps, because of a mild thermal emission contribution at the GOES range. Accordingly, the low-energy cutoff was fixed to $10 \mathrm{keV}$ (i.e. below the Konus- Wind fitting range). The mean atomic number of the target plasma, $Z$, was set to 1.2 to account the contribution from target nuclei heavier than hydrogen.

The fit examples are given in Figure 3 while the fit results are summarized in Figure 4 and in Table 1 (the errors are given at the $68 \%$ confidence level). The fact that the HXR spectral analysis could only be performed for a few uneven time intervals \#1-8 indicated in Fig. 1 $1 \mathrm{~d}$ complicates the study of the spectral evolution and comparison with the microwave spectral data. However, if the actual electron spectrum does not deviate strongly from a single power-law, the effective spectral slope can be estimated from the hardness ratio, which is the ratio of $\mathrm{X}$-ray fluxes recorded in two adjacent wide energy ranges, G1 and G2, or G2 and G3. To this end we fitted the spectra in our eight available intervals with a single power-law (brmThick) at the photon energy range 20-200 keV and then cross-correlated the obtained spectral index 

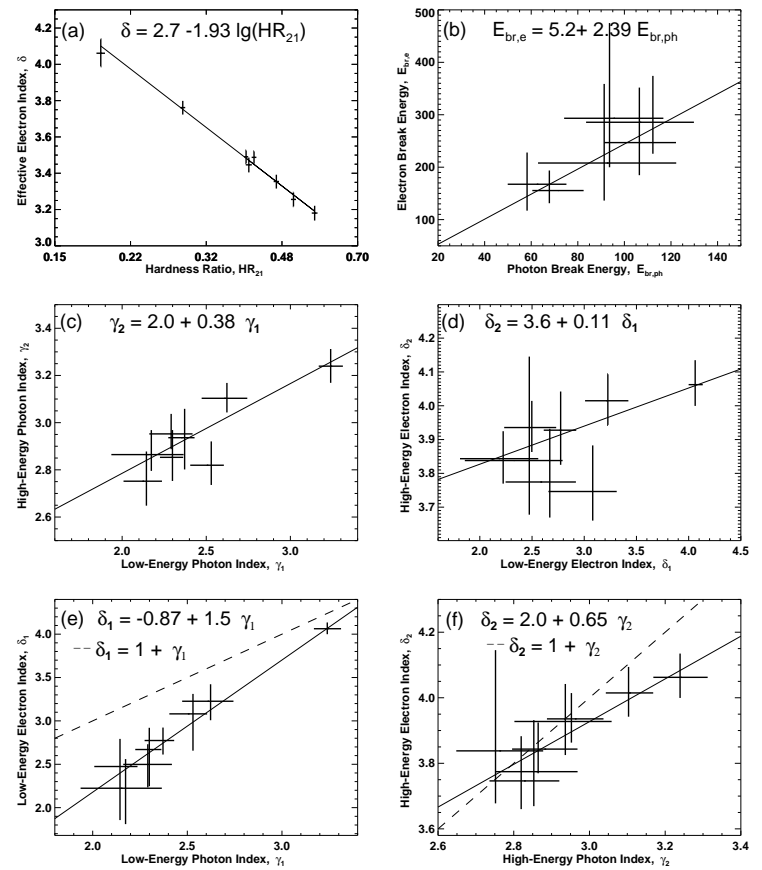

FIG. 4.- Correlation plots for the HXR fit parameters. (a) Cross-correlation between effective power-law index of electron distribution $\delta$, derived from the brmThick fit, Eq. (2), and hardness ratio $\mathrm{HR}_{21}$ for the eight analyzed spectra; (b) crosscorrelation between the break energies in the photon and electron fits; (c) cross-correlation between the low- and high- energy photon indices $\gamma_{1,2}$; (d) cross-correlation between the lowand high- energy electron indices $\delta_{1,2}$; (e) cross-correlation between the photon and electron low-energy indices $\gamma_{1}$ and $\delta_{1}$; (f) cross-correlation between the photon and electron high-energy indices $\gamma_{2}$ and $\delta_{2}$. The dashed lines in (e) and (f) show simple expectation based on classical thick-target model $\delta=\gamma+1$.

$\delta$ with the hardness ratio $H R_{21}$. Figure 4 a shows that these two values demonstrate excellent correlation and reveal the following regression law:

$$
\delta=2.7-1.93 \log H R_{21},
$$

thus, the spectral evolution in this energy range can be recovered with a very high cadence: since the data of the spectral hardness are available with a very high time resolution (down to $16 \mathrm{~ms}$ ) the $\delta$ time evolution can be recovered with a comparably high time resolution. We looked but did not find any significant variation of the spectral index $\delta$ on the time scales much shorter than $1 \mathrm{~s}$ and; thus, we derived the $\delta$ evolution with $0.5 \mathrm{~s}$ cadence needed for comparison with the microwave spectral fit results available with the same cadence.

As has been said, the single power-law does not fit well the data in the entire range $20-1000 \mathrm{keV}$; therefore, we used a broken power-law over either photon or electron energy. We found the results of the fitting to the photon power-law (BPL) are more stable as compared with the fit to the electron power-law (brmThick2): in the latter case the confidence interval of the fit parameters appears to be rather broad if all the parameters of the broken power-law are kept free, especially, for intervals 4-7 (again, we are referring to Fig. 11), while interval 8 can, in fact, be fit by a single power-law model. This behavior of the fit implies that the change of the spectral slope is modest and likely gradual, so no exact value of the break energy could be identified from the data. To get a more stable fit results for intervals $4-7$, we first fixed the high-energy slope $\delta_{2}$ to the values implied by the BPL fits $\left(\delta_{2}=\gamma_{2}+1\right)$ and determined the break energies in the electron spectrum. Then, we cross-correlated the break energies in these two fits, BPL and brmThick2, to determine a regression law between them, Figure $4 \mathrm{~b}$. At the next step we fixed the break energies at the values implied by this regression law and fit the spectra with free spectral indices. The results of these slightly different versions of the brmThick2 fits agree well to each other, which confirms that the spectral model with a broken power-law is overall consistent with the data.

The remaining panels of Figure 4 display correlation plots between different pairs of the spectral indices obtained from the fits. Figure $4 \mathrm{k}, \mathrm{d}$ show correlation plots between the low- and high- energy spectral indices. The results are somewhat different between the BPL and brmThick2 fits. Although for the BPL fit $\gamma_{1}$ and $\gamma_{2}$ show a significant correlation, the correlation between $\delta_{1}$ and $\delta_{2}$ is barely visible, implying that the low- and high- energy slopes in the spectrum of nonthermal electrons are independent. If so, the correlation between $\gamma_{1}$ and $\gamma_{2}$ can be understood as a result of some contribution of high-energy electrons into the low-energy photon spectrum.

The classical thick-target model (Brown 1971; Somov \& Syrovatskii 1976) implies a simple unique relationship between the photon and electron spectral indices, $\delta=\gamma+1$. However, one can note that the numbers in Table 1 do not follow this simple formula because additional physical processes are included in brmThick2 model and also because of potential complexity of the spectrum, which is not captured by the simplified models. The two bottom panels of Figure 4 display the correlation plots between the low-energy $\left(\gamma_{1}\right.$ and $\left.\delta_{1}\right)$ and high-energy $\left(\gamma_{2}\right.$ and $\left.\delta_{2}\right)$ spectral indices, respectively. In both cases there is a significant correlation, which, however, noticeably deviates from the expectation based on the classical thick-target emission model. Interestingly, for the high-energy spectral indices the regression curve is shallower than $\delta_{2}=\gamma_{2}+1$ (dashed line) although most of the index pairs are quantitatively consistent with the expectation of $\delta_{2}=\gamma_{2}+1$. In contrast, for the low-energy indices the regression is steeper and does not cross the data points at all, which can again be interpreted in terms of contribution from the high-energy electrons to the lower-energy X-ray emission.

The spectra are hard in the entire considered energy range. The low-energy part of the spectrum shows $\gamma_{1} \sim 2-3.3$ and $\delta_{1} \sim 2-4$. The steeper high-energy spectra are still hard with $\gamma_{2} \sim 2.7-3.3$ and $\delta_{2} \sim$ $3.7-4.1$. The two last spectra, 7 and 8 , measured at the decay phase of the flare, have two peculiarities: a dip at $\simeq 250 \mathrm{keV}$ and a systematic excess of emission above $\simeq 500 \mathrm{keV}$ as compared to the model.

The fluence of the flare measured from 01:34:54.105 UT to 01:35:13.817 UT is $(1.214 \pm 0.020) \times$ $10^{-4} \mathrm{erg} \mathrm{cm}^{-2}$ and the $64 \mathrm{~ms}$ peak flux measured from 01:35:00.761 UT to 01:35:01.273 UT is $(1.95 \pm 0.05) \times$ 
$10^{-5} \mathrm{erg} \mathrm{cm}^{-2} \mathrm{~s}^{-1}$ (both in the $20-1000 \mathrm{keV}$ range). Assuming isotropic emission, the corresponding radiated X-ray energy is $(3.36 \pm 0.06) \times 10^{23} \mathrm{erg}$ and the peak HXR luminosity is $(5.40 \pm 0.13) \times 10^{22} \mathrm{erg} \mathrm{s}^{-1}$.

\subsubsection{Microwave Spectra}

To obtain the microwave spectral evolution we employed two synthetic dynamic spectra, 'slow' (Figure 1 $\mathrm{b}$ ) and 'fast' (Figure 1), described in Section 2.1. The slow dynamic spectrum allows for longer tracking the burst evolution, while the fast one has an advantage of having a better time resolution and $80 \mathrm{GHz}$ data that generally help to better constrain the high-frequency spectral slope. It has to be kept in mind, however, that if the spectral peak is too high $(\sim 35 \mathrm{GHz})$ and the high-frequency slope is only constrained by the $4 \mathrm{~s}$ smoothing of the poorly defined $80 \mathrm{GHz}$ data point (see Figure 2r), the value of high-frequency microwave spectral index could not be determined reliably.

Both slow and fast dynamic spectra were sequentially fitted with a so-called microwave generic function proposed by Stahli et al. (1989):

$$
S=e^{A} f^{\alpha}\left[1-e^{-e^{B} f^{-\beta}}\right]
$$

where $f$ is the frequency in $\mathrm{GHz}, A, B, \alpha$, and $\beta$ are the free fitting parameters, from which the relevant spectral parameters are computed. Specifically, the low-frequency spectral index $\alpha_{\mathrm{lf}} \equiv \alpha$, while the highfrequency spectral index is $\alpha_{\mathrm{hf}}=\alpha-\beta$. The peak frequency and the flux density at peak frequency are calculated from the shape of function $S$ as described by Nita et al. (2004). Following Nita et al. (2004) we used the corresponding built-in functionality of the ovsa_explorer widget from the OVSA software available from the SSW distribution.

Figure 5 shows a summary of the microwave spectral fit. The fit results obtained from the slow and fast dynamic spectra generally agree to each other, but nevertheless show a number of mismatches; especially, during the highly impulsive peak phase. The peak flux and peak frequency determined from the fast dynamic spectrum display a significantly stronger variation than the slow ones, which is real and reflects actual subsecond impulsiveness of the burst. On the other hand, the corresponding strong variations of the 'fast' high-frequency spectral index are not real: they only take place when the spectral peak frequency is about $35 \mathrm{GHz}$, so that the high-frequency slope is only constrained by poorly known $80 \mathrm{GHz}$ data, as pointed out above.

There are a number of properties of the fit parameter evolution, which are noteworthy to mention. The spectral peak frequency demonstrates a remarkably large variation far more than by an order of magnitude: it is around $10 \mathrm{GHz}$ at the burst rise phase, then it goes up to at least $35 \mathrm{GHz}$ during the peak phase, and finally decreases down to roughly $1.5 \mathrm{GHz}$ at the decay phase. Thus, the entire range of the spectral peak variation is within a factor larger than 20 - a substantially broader range than for a 'typical' microwave burst (Nita et al. 2004; Melnikov et al. 2008). This observation alone is a
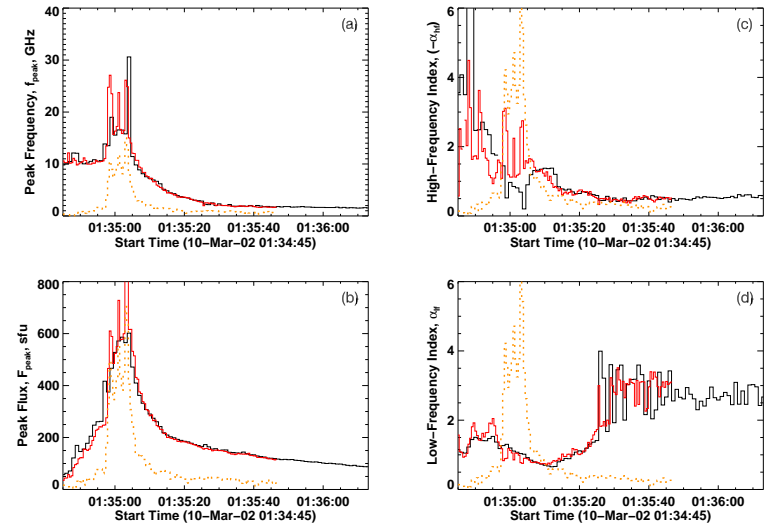

FiG. 5.- Microwave spectral fit parameter evolution: black curves - from $1 \mathrm{~s}$ data, red curves - from $0.5 \mathrm{~s}$ data, dotted orange curves are the appropriately scaled light curve at $35 \mathrm{GHz}$. (a) peak frequency evolution. (b) peak flux evolution. (c) evolution of high-frequency spectral index. (d) evolution of lowfrequency spectral index.

strong evidence that the magnetic field at the radio source at the decay phase is much smaller than that at the peak phase; see Section 2.5 for details. Although there is an overall correspondence between the spectral peak flux and peak frequency in the sense that the larger the flux the larger the peak frequency, there is no perfect correlation between these two parameters. Indeed, if we compare the rise and decay phases, we note immediately that the same peak flux corresponds to substantially smaller peak frequency at the decay than at the rise phase. Note also, that at the early decay phase the peak flux and frequency decline highly consistently, while after roughly 01:35:30 UT the decrease of the peak frequency terminates although the peak flux continues to decline.

The high- and low- frequency spectral indices also display a substantial evolution. At the rise, peal 8 , and early decay phases the high-frequency spectral index shows a soft-hard-soft (SHS) pattern similar to that often reported for HXR spectra. But then, around 01:35:10 UT, the softening terminates and again gives a way to the spectral hardening. This hardening continues until 01:35:30 UT, when $\alpha_{\mathrm{hf}}$ hits the level of $\alpha_{\mathrm{hf}} \approx-1$ and than stays roughly constant. The low-frequency spectral index decreases all the way during the rise, peak, and early decay phases until roughly 01:35:10 UT and then turns to increase until 01:35:30 UT, when it hits the level of $\alpha_{\mathrm{lf}} \approx 3$. After that it stays approximately constant at this level until the end of the event. Overall, the event demonstrates a strikingly prominent spectral variability over the rise, peak, and early decay phases (until 01:35:30 UT), while, in contrast, shows no spectral evolution after that.

\subsubsection{Comparison of the X-ray and Microwave Spectral Indices}

Having both HXR and microwave spectral fits it is reasonable to compare the 'effective' spectral index $\delta$ of the electron flux derived from the HXR hardness ratio with Eq. (4) and the high-frequency microwave

\footnotetext{
8 Neglecting the three outliers during three most impulsive peaks.
} 
TABLE 1

Summary of the Konus- Wind spectral fits (20 KeV - $1 \mathrm{MeV}$ )

\begin{tabular}{ccccccccc}
\hline \hline No. & $\begin{array}{c}\mathrm{T}_{\text {start }}^{\mathrm{a}} \\
(\mathrm{s})\end{array}$ & $\begin{array}{c}\Delta \mathrm{T} \\
(\mathrm{s})\end{array}$ & $\gamma_{1}$ & $\gamma_{2}$ & $\begin{array}{c}E_{b r, p h} \\
\mathrm{keV}\end{array}$ & $\begin{array}{c}\text { BPL } \\
\mathrm{A}^{\mathrm{b}}\end{array}$ & Flux $^{\mathrm{c}}$ & $\chi^{2} / \mathrm{dof}^{2}$ \\
\hline 1 & 0.000 & 5.632 & $2.53_{-0.12}^{+0.08}$ & $2.82_{-0.08}^{+0.10}$ & $91_{-28}^{+30}$ & $0.079_{-0.004}^{+0.008}$ & $4.48_{-0.13}^{+0.13}$ & $0.67(36.7 / 55)$ \\
2 & 5.632 & 1.024 & $2.30_{-0.07}^{+0.06}$ & $2.85_{-0.10}^{+0.11}$ & $106_{-15}^{+15}$ & $0.268_{-0.011}^{+0.014}$ & $13.3_{-0.3}^{+0.3}$ & $0.86(47.4 / 55)$ \\
3 & 6.656 & 0.512 & $2.14_{-0.14}^{+0.09}$ & $2.75_{-0.10}^{+0.13}$ & $94_{-19}^{+23}$ & $0.424_{-0.028}^{+0.048}$ & $19.5_{-0.5}^{+0.5}$ & $0.82(29.4 / 36)$ \\
4 & 7.168 & 1.024 & $2.37_{-0.10}^{+0.06}$ & $2.94_{-0.13}^{+0.12}$ & $112_{-29}^{+17}$ & $0.232_{-0.009}^{+0.017}$ & $11.9_{-0.3}^{+0.3}$ & $1.1(60.5 / 55)$ \\
5 & 8.192 & 1.024 & $2.29_{-0.13}^{+0.13}$ & $2.95_{-0.06}^{+0.08}$ & $68_{-7}^{+14}$ & $0.33_{-0.03}^{+0.04}$ & $14.6_{-0.4}^{+0.4}$ & $0.87(47.8 / 55)$ \\
6 & 9.216 & 0.512 & $2.17_{-0.24}^{+0.19}$ & $2.86_{-0.07}^{+0.10}$ & $58_{-8}^{+17}$ & $0.48_{-0.08}^{+0.12}$ & $18.8_{-0.6}^{+0.6}$ & $0.71(24.8 / 35)$ \\
7 & 9.728 & 1.792 & $2.62_{-0.15}^{+0.12}$ & $3.10_{-0.06}^{+0.07}$ & $58_{-7}^{+8}$ & $0.186_{-0.027}^{+0.018}$ & $9.9_{-0.3}^{+0.3}$ & $1.15(47.0 / 41)$ \\
$8^{\mathrm{f}}$ & 11.520 & 8.192 & $3.24_{-0.07}^{+0.07}$ & $3.24_{-0.07}^{+0.07}$ & $\ldots$ & $0.0200_{-0.0010}^{+0.0010}$ & $2.21_{-0.20}^{+0.20}$ & $0.82(46.8 / 57)$
\end{tabular}

TABLE 1

(CONTINUED)

\begin{tabular}{cccccccc}
\hline \hline No. & $\begin{array}{c}\mathrm{T}_{\text {start }}{ }^{\mathrm{a}} \\
(\mathrm{s})\end{array}$ & $\begin{array}{c}\Delta \mathrm{T} \\
(\mathrm{s})\end{array}$ & $\delta_{1}$ & $\delta_{2}$ & $\begin{array}{c}\text { brmThick2 } \\
E_{b r, e} \\
\mathrm{keV}\end{array}$ & $\begin{array}{c}\text { Electron } \\
\text { flux }\end{array}$ & $\chi^{2} /$ dof \\
\hline 1 & 0.000 & 5.632 & $3.08_{-0.42}^{+0.23}$ & $3.75_{-0.09}^{+0.14}$ & $208_{-72}^{+151}$ & $1.0_{-0.5}^{+0.6}$ & $0.66(36.5 / 55)$ \\
2 & 5.632 & 1.024 & $2.67_{-0.42}^{+0.25}$ & $3.77_{-0.11}^{+0.16}$ & $247_{-62}^{+105}$ & $1.1_{-0.6}^{+0.8}$ & $0.92(50.6 / 55)$ \\
3 & 6.656 & 0.512 & $2.47_{-0.62}^{+0.32}$ & $3.84_{-0.16}^{+0.31}$ & $293_{-93}^{+182}$ & $1.0_{-0.6}^{+0.9}$ & $0.84(30.2 / 36)$ \\
4 & 7.168 & 1.024 & $2.77_{-0.16}^{+0.15}$ & $3.93_{-0.10}^{+0.11}$ & $282^{\mathrm{e}}$ & $1.3_{-0.4}^{+0.5}$ & $1.1(61.0 / 56)$ \\
5 & 8.192 & 1.024 & $2.50_{-0.26}^{+0.23}$ & $3.94_{-0.07}^{+0.08}$ & $169^{\mathrm{e}}$ & $1.3_{-0.5}^{+0.7}$ & $0.88(49.2 / 56)$ \\
6 & 9.216 & 0.512 & $2.2_{-0.4}^{+0.3}$ & $3.84_{-0.07}^{+0.08}$ & $144^{\mathrm{e}}$ & $1.1_{-0.5}^{+0.9}$ & $0.72(26.0 / 36)$ \\
7 & 9.728 & 1.792 & $3.23_{-0.22}^{+0.20}$ & $4.01_{-0.07}^{+0.03}$ & $144^{\mathrm{e}}$ & $4.3_{-1.5}^{+2.0}$ & $1.12(47.1 / 42)$ \\
$8^{\mathrm{f}}$ & 11.520 & 8.192 & $4.07_{-0.07}^{+0.07}$ & $4.07_{-0.07}^{+0.07}$ & $\ldots$ & $4.1_{-0.6}^{+0.8}$ & $0.81(46.4 / 57)$
\end{tabular}

${ }^{a}$ Since $t_{0}(\mathrm{KW})=5694.105 \mathrm{~s}$ UT (01:34:54.105).

b In units of photons $\mathrm{cm}^{-2} \mathrm{~s}^{-1} \mathrm{keV}^{-1}$.

${ }^{\text {c }}$ In units of erg $10^{-6} \mathrm{~cm}^{-2} \mathrm{~s}^{-1}$ in energy range $20-1000 \mathrm{keV}$.

d In units of electrons $10^{35} \mathrm{~s}^{-1}$.

e Fitted with frozen $E_{b r, e}$.

${ }^{\mathrm{f}}$ Fitted by a single power law.

spectral index $\alpha_{\text {hf }}$ primarily defined by the energy spectrum of the fast electron number density in the source of the microwave emission. Figure [6] shows that these spectral indices evolve consistently over the impulsive phase of the event as marked at the $\delta$ curve by plus signs outside the mentioned outliers. Both spectral indices show the SHS evolution, while they tend to disagree outside the impulsive peak. In spite of this apparent consistency, the scatter plot of the spectral indices during the impulsive phase displays no perfect correlation, Figure $6 \mathrm{~b}$, although the data points roughly follow the linear regression law $\delta \approx$ $2.3-0.98 \alpha_{\mathrm{hf}}$.

\subsection{Imaging}

A summary of the imaging data is given in Figure 7 The background color in Figure 7 left, shows one of the two $195 \AA$ EIT/SOHO difference image: 9 on which a representative set of microwave contours is superimposed along with the relevant neutral lines

9 Three $195 \AA$ images were available taken on 01:25:52.555 UT, 01:36:14.749 UT, and 01:48:06.640 UT.
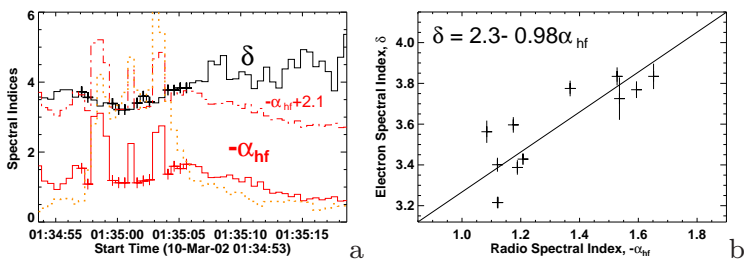

FIG. 6.- Comparison between the microwave and HXR spectral indices. (a) evolution of the electron flux spectral index $\delta$ (black curve) derived from the spectral hardness as explained in Section 2.3.1 and microwave high-frequency spectral index $\alpha_{\text {hf }}$ (red curve). The red dash-dotted curve shows the same microwave spectral index but displaced by 2.1 up to ease the visual comparison with the HXR-derived electron spectral index. The plus symbols show the data points used for correlation analysis during the impulsive flare phase, which is envisioned by the $35 \mathrm{GHz}$ light curve shown in orange dotted curve. (b) the scatter plot of the indices and the corresponding linear fit.

obtained from the SoHO/MDI photospheric magnetogram.

The difference image demonstrates the presence of two bright compact kernels at the flare time separated by about $10^{\prime \prime}$ in the North-South direction, which are not seen 12 minutes apart either before or after the 

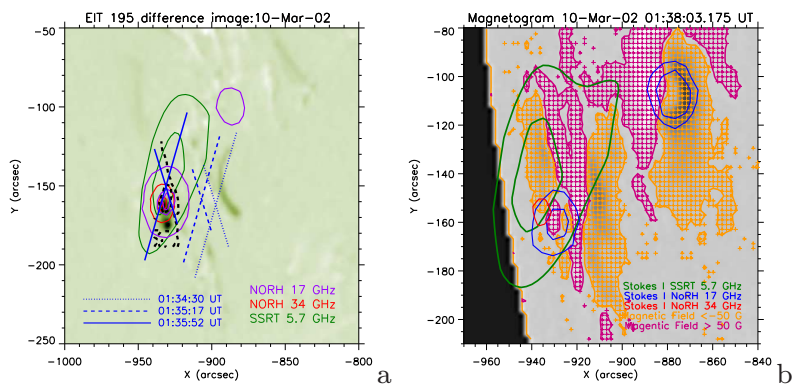

FIG. 7.- (a) The $195 \AA$ EIT/SOHO difference negative image between 01:36:14.7490 UT and 01:25:52.555 UT. Contours show microwave images at $34 \mathrm{GHz}$ (red) and $17 \mathrm{GHz}$ (violet) obtained at 01:35:01 UT by NoRH with intensity levels at $20 \%$ and $70 \%$ of the maximum. Green contours show $5.7 \mathrm{GHz}$ image obtained by SSRT at 01:37:11 UT with intensity levels $70 \%$ and $90 \%$ of maximum. The dashed black line shows several neutral lines taken from photospheric magnetogram by MDI/SOHO. Intersections of the blue cross bars (dotted, dashed, and solid) present centroids of $5.7 \mathrm{GHz}$ source at different moments. The direction of each cross bar shows the scanning direction of either EW or NS array. The length of each cross bar indicates the source width at half maximum over this bar direction. (b) SoHO/MDI magnetogram, where the locus of pixels with $B_{\|}>50 \mathrm{G}$ is shown in magenta, while with $B_{\|}<-50 \mathrm{G}$ is shown in orange. Contours of the microwave emission at 5.7, $17, \& 34 \mathrm{GHz}$ are shown for the decay phase.

flare. Microwave images at 17 and $34 \mathrm{GHz}$ also show a double source structure but with a much larger separation between the sources, roughly $85^{\prime \prime}$. It is interesting that the EUV kernels are co-located with the strongest, southern, microwave source; the northern kernel spatially coincides with the centroid of the microwave source at the impulsive flare phase. There are two magnetic neutral lines; the shorter one separates the EUV kernels, while the longer one separates the microwave sources. These relationships imply that a magnetic connectivity is possible both between the EUV kernels and between the microwave sources.

The positions of the 17 and $34 \mathrm{GHz}$ sources do not change at the course of the flare. At the impulsive phase the southern source dominates at both 17 and $34 \mathrm{GHz}$ (Figure 7), while at the decay phase the brightness of these two sources is comparable at $17 \mathrm{GHz}$; however, the northern source is not seen at all at $34 \mathrm{GHz}$. Both microwave sources produce left circular polarization (LCP) at $17 \mathrm{GHz}$. Remarkably (Figure 8), the Northern source has a very strong degree of polarization, which reaches up to $80 \%$ at the beginning of the rise phase and remains strong afterwards, $\sim 60 \%$. In contrast, the degree of polarization of the stronger, Southern source is much weaker being about $20 \%$ on average, but is strongly reduced during the impulsive phase, when the $34 \mathrm{GHz}$ emission has the strongest intensity. The spatially resolved microwave light curves display a prominent time delay between the southern and the northern sources, which implies that the electron acceleration takes place at or close to the southern source, while the electrons reach the remote northern source only after traveling roughly $2 \mathrm{~s}$ through a coronal loop.

The first 2D image at $5.7 \mathrm{GHz}$ is available at 01:37:11 UT, i.e., already at the decay phase. There is one single source which is displaced compared with any of the high-frequency sources in a way implying a

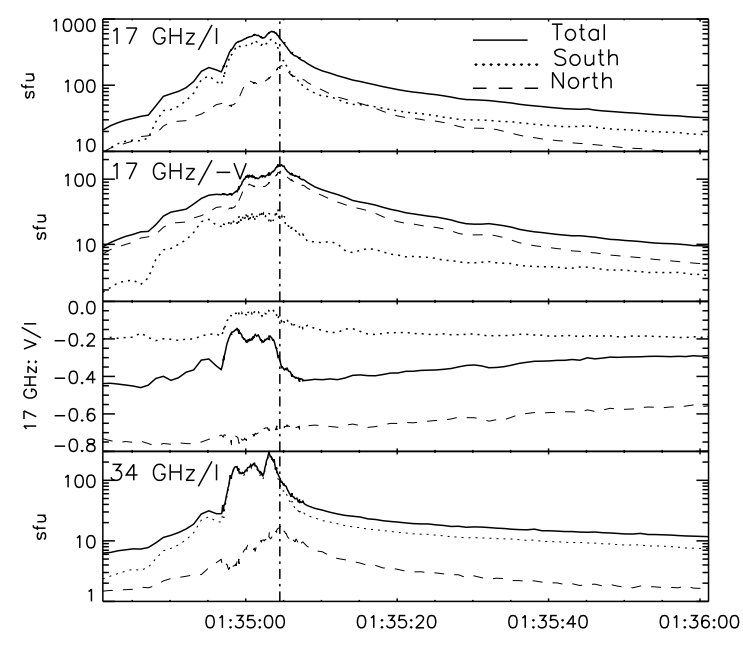

FIG. 8.- NoRH light curves: (a) flux densities from the north and south sources along with the integrated data at $17 \mathrm{GHz}$; (b) the same for the Stokes V parameters; (c) the same for degree of polarization; and (d) the flux densities at $34 \mathrm{GHz}$.

loop-like connectivity between the NoRH sources; its centroid is located closer to the southern than to the northern NoRH source. The $5.7 \mathrm{GHz}$ source evolves noticeably during the flare. Given that SSRT produces only one 2D map per 2-3 minutes, we employ 1D SSRT scans to study this evolution. The intersections of the dotted, dashed, and solid blue lines in Figure 7 (from right to left) show the locations of the $5.7 \mathrm{GHz}$ source centroid before, at, and after the impulsive phase of the event, respectively. Apparently, the source centroid moves eastward with the velocity estimated as $3.2 \times 10^{7} \mathrm{~cm} / \mathrm{s}$ during the rise, peak, and early decay phases and then stops moving such as its position is almost precisely the location of the 2D image taken at 01:37:11 UT. The apparent source sizes remain roughly constant during the burst being about $50^{\prime \prime} \times 100^{\prime \prime}$, which implies the true sizes to be about $40^{\prime \prime} \times 90^{\prime \prime}$ or less.

\subsection{Summary of the Data}

There are two striking features in the flare under study. The first one is a prominent variation of the time profile impulsiveness - from highly impulsive HXR and high-frequency microwave light curves (particularly, at $35 \mathrm{GHz}$ ) - to more and more gradual microwave light curves at progressively lower frequencies and the SXR light curves. This makes it difficult to think of this event in terms of the conventional Neupert effect. Indeed, Figure 2 2 d,e show that the light curves at $35 \mathrm{GHz}$ or, equivalently, the HXR ones, do not correlate with the SXR time derivative, but correlate tightly with the derivative of the microwave light curve at $3.75 \mathrm{GHz}$. This correlation could imply that the $3.75 \mathrm{GHz}$ light curve represents the thermal response of the heated plasma. However, the SXR time derivative correlates well with the $3.75 \mathrm{GHz}$ light curve itself, which would instead imply that the $3.75 \mathrm{GHz}$ light curve is the most representative one for the nonthermal particle impact. Thus, 
the classification of the light curves onto thermal and nonthermal ones based solely on their impulsiveness becomes inconclusive in our event, which calls for a more detailed analysis of the observed relationships.

The second striking property, closely related to the first one, is a remarkable spectral evolution of the microwave burst. During the rise phase and the impulsive peak the microwave spectrum displays a conventional (inverted bell-shaped) form with the spectral peak frequency well correlated with the peak flux (cf., Nita et al. 2004). This correlation is indicative of the optical thickness effect in the spectral peak formation (Dulk 1985; Melnikov et al. 2008). Then, at a later, gradual phase the spectrum becomes essentially flat between 1 and $10 \mathrm{GHz}$. Overall, the spectral evolution can be characterized as a progressive low-frequency flattening over the course of the flare. This flattening can be understood if the microwave source is getting more and more nonuniform with time. However, this is only a part of the puzzle: over the gradual phase the degree of polarization is unexpectedly large at $3.75-5.7 \mathrm{GHz}$ as well as at $17-$ $35 \mathrm{GHz}$ indicative of optically thin emission at these spectral ranges, while small at $1-2 \mathrm{GHz}$ and $9.4 \mathrm{GHz}$ as expected for the optically thick emission. This implies that two distinct nonuniform sources (presumably, loops) are involved.

\section{MODELING}

The first of the two loops implied by the data, no doubts, produces nonthermal emission that dominates the impulsive component at high frequencies. The second loop produces a gradual emission, which can be either thermal or nonthermal, that dominates at the lower frequencies. We carefully addressed the question if this gradual emission is thermal or nonthermal and rejected the thermal model; see Appendix $\mathrm{A}$

\subsection{Nonthermal model for the gradual flare component}

Given that we rejected the thermal model of the gradual emission component in the given flare we turn now to a nonthermal model. The most challenging for a nonthermal model is to explain, why the lowerfrequency light curves have a longer decay constant than the higher-frequency ones; the property which holds all the way from $35 \mathrm{GHz}$ down to $0.6 \mathrm{GHz}$. Indeed, the extended microwave emission is commonly ascribed to the fast electron fraction trapped in the coronal part of the flaring loop (often, the looptop) due to magnetic mirroring effect (e.g., Melnikov 1994; Melnikov \& Magun 1998; Bastian et al. 1998; Lee \& Garv| 2000; Lee et al. 2000; Kundu et al. 2001; Melnikov et al. 2002, and many others). Then, the fast electrons lose their energy due to Coulomb collisions. They are also scattered into the loss-cone and are getting lost from the loop due to the Coulomb scattering. The characteristic time constants of the energy loss and angular scattering due to Coulomb collisions both increase with the electron energy; so the high-energy electrons survive longer in the coronal trap than the lower-energy electrons. The higherenergy electrons radiate at higher frequency; thus, the higher-frequency microwave emission is supposed to have a longer decay time than the lower-frequency emission. This behavior has been observed in many cases, but our event displays an exactly opposite trend.

To understand the likely causes of this unusual trend we refer to a seminal paper by Lee et al. (1994), who thoroughly studied four X-class flares with unusually flat microwave spectra in the $1-20 \mathrm{GHz}$ range; the X9.4 1991-Mar-22 flare from their sample demonstrates the closest resemblance to our event in terms of the light curve duration vs frequency. Lee et al. (1994) undertook a simplified 3D modeling with a dipole magnetic loop to interpret the observed properties of the microwave emission in their event sample. They found that for a dipole loop, which is big and nonuniform (has a reasonably high mirror ratio), the microwave spectrum can be remarkably flat in a rather broad spectral range, even from 1-20 GHz for sufficiently large magnetic loop. This conclusion is confirmed by a more sophisticated 3D modeling reported by Kuznetsov et al. (2011).

In addition to the interpretation of the flat spectra at the flare peak phase, Lee et al. (1994) also offered an elegant and convincing scenario for the flare evolution, which naturally results in the observed 'anomalous' behavior of the microwave light curves vs frequency. Originally, at the impulsive rise phase, the microwave source is relatively compact and occupies a volume with a relatively strong magnetic field, which results in high spectral peak frequency, $f_{\text {peak }} \gtrsim 20 \mathrm{GHz}$. Then, the radio source expands to occupy a much bigger nonuniform loop10, and produces the flattest spectrum at the phase, when the source becomes the most nonuniform (i.e., magnetic field varies over the broadest range of values). Later, at the decay phase, the radio source 'shrinks' toward the looptop, where the magnetic field is low, thus, resulting in a decrease of the spectral peak frequency toward $1 \mathrm{GHz}$ at the decay phase. Lee et al. (1994) noted that the fast electron spectral softening (with the electron spectral index $\delta$ change from around 3 at the peak phase to 4 at the decay phase) can further improve the consistency of the model microwave spectral evolution with the observed one. It is highly likely that a very similar scenario happened in our event in spite of the fact that it is only a C5.1 GOES class flare, i.e., two orders of magnitude smaller than the X-class flares analyzed by Lee et al. (1994).

\subsection{The flare morphology suggested by the data.}

Locations of and relationships between various EUV and microwave sources suggest that this flare belongs to Hanaoka morphological type (Hanaoka 1997), where the flare energy release is believed to be driven by interaction between a small compact loop, whose footpoints are highlighted by the EUV kernels, and a bigger loop, whose footpoints are highlighted by the high-frequency microwave sources at 17 and $34 \mathrm{GHz}$, while the coronal part of the loop is implied

\footnotetext{
10 Lee et al. (1994) proposed an 'inflated' magnetic loop, but a similar effect can be achieved if the fast electrons gradually fill bigger and bigger fraction of a large magnetic trap.
} 
by the $5.7 \mathrm{GHz}$ image. Remarkably, this two-loop configuration is supported by other available data. For example, there are neutral lines both between the EUV kernels and between the microwave footpoints, which indicates that the corresponding magnetic connectivities are likely.

Independent evidence in favor of two distinct sources comes from the microwave polarization spectrum. Indeed the degree of polarization is very small at $1-2 \mathrm{GHz}$ indicating optically thick emission at these frequencies, but turns high at $3.75-5.7 \mathrm{GHz}$ manifesting optically thin emission here. However, the degree of polarization is low again at $9.4 \mathrm{GHz}$, while once again high at $17-35 \mathrm{GHz}$. This behavior of the degree of polarization is entirely inconsistent with a single (even though spatially nonuniform) source (Kuznetsov et al. 2011), while requires two distinct radio sources with strongly differing magnetic fields.

It is reasonable to assume that a smaller loop has a larger magnetic field, while a larger loop has a weaker magnetic field and, therefore, can form an efficient magnetic trap. The microwave light curves support this idea. Indeed, the high-frequency light curve at $35 \mathrm{GHz}$, which is supposed to originate from a source with the strongest magnetic field, is highly impulsive (as well as all HXR light curves) and does not show any evidence of the fast electron trapping in a coronal loop. Thus, it is likely formed in the small loop with strong magnetic field and small mirror ratio, which makes the magnetic trapping inefficient. In contrast, the lower-frequency optically thin light curve at $3.75 \mathrm{GHz}$ has a delayed tail such as if the fast electrons were injected from the small loop (or from an interaction region of these two loops) and then accumulated in the bigger loop. This casual relationship is further supported by close correlation between the time derivative of the gradual $3.75 \mathrm{GHz}$ light curve and the impulsive $35 \mathrm{GHz}$ and HXR light curves.

\subsection{Validating the model with $3 D$ modeling}

Even though the outlined flare model seems plausible, the current state-of-the-art requires that it is quantitatively validated by a 3D modeling based on appropriate magnetic extrapolation as in Fleishman et al. (2011, 2013, 2016); Nita et al. (2015); Kuznetsov \& Kontar (2015). However, the modeling is substantially complicated in our case for the following reasons. Since two different loops with presumably different twists are involved in the flaring process, it is unlikely that they could be reproduced within either potential or linear force-free field (LFFF) extrapolation used in the cited studies addressing a single loop only, so a nonlinear force-free field (NLFFF) extrapolation is called for. However, there is no vector magnetogram available to perform NLFFF extrapolation. We do have the line-of-sight magnetic data from SoHO/MDI, which is formally sufficient to produce a LFFF extrapolation, but it will necessarily be imperfect since the region of interest is located very close to the limb11. This implies

\footnotetext{
11 Typically, a model built from a LFFF extrapolation close to the limb requires that the model magnetic field is scaled by
}

that we can only perform a number of tests with the available data, but not a comprehensive modeling. In particular, we are forced to create two separate LFFF models, with presumably different force-free parameters $\alpha$ - one for each of the two loops involved in our flare.

With this reservation, we are going to employ the powerful GX Simulator tool (Nita et al. 2015) to test if there is a model (a set of two different data cubesone for each loop) consistent with photospheric magnetogram that can answer the following key questions about the event:

1. If the implied small and big loops can be reproduced in LFFF extrapolated data cubes and what $\alpha$ are needed for that?

2. Is it possible to populate the small loop with a distribution of fast electrons, which is consistent with the HXR data and, at the same time, capable of reproducing the high-frequency microwave spectrum?

3. Is it possible to populate the big loop with a distribution of fast electrons consistent with the HXR data to reproduce the low-frequency microwave spectrum?

4. Could the entire spectrum be reproduced by the two-loop model?

5. Is it possible to get the LCP polarization from both $17 \mathrm{GHz}$ sources?

6. Is it possible to get a very high degree of LCP polarization from the north $17 \mathrm{GHz}$ source?

7. Could the entire polarization spectrum be reproduced by the two-loop model?

Let us start from a model needed to reproduce the small loop. After a number of trials and errors (cf. Fleishman et al. 2016) with the built-in LFFF engine of GX Simulator (Nita et al. 2015), we obtained a narrow range of $\alpha \approx-1.75 \times 10^{-9} \mathrm{~cm}^{-1}$ with which the connectivity between the EUV kernels can be reproduced as shown in Figure 9. The central field line (shown in red) has a length of $L_{\text {small }} \approx 8.84 \times 10^{8} \mathrm{~cm}$, the magnetic field value at the loop top ${ }^{12} B_{\text {small,lt }} \approx$ $620 \mathrm{G}$, and the mirror ratio less than two.

For microwave spectral modeling we select the peak time at 01:35:03.600 UT. One of the key ingredients for the modeling is the shape of the distribution function of the nonthermal electrons. Although the microwave data themselves can be successfully fit by a single power-law distribution of fast electrons over energy 13 we adopt here a broken power-law as suggested by the Konus-Wind X-ray fit (cf. Table 11).

a small number.

12 A scaling factor of 3 has been applied to the originally extrapolated magnetic data cube, (cf. Fleishman et al. 2016).

13 This is because the microwave spectrum at these high frequencies is not sensitive to the exact shape of the nonthermal electron spectrum at low energies, where the break of this spectrum is suggested by the Konus-Wind data. This results in a well-known uncertainty while estimating energy contents and other measures determined by the low-energy part of the nonthermal electron spectrum. 
We get a reasonably good spectral match at high microwave frequencies if we populate this magnetic loop with fast electron distribution (cf. Table 1 intervals 5-6) with $E_{\min }=10 \mathrm{keV}, E_{\mathrm{br}}=150 \mathrm{keV}, E_{\max }=$ $1.8 \mathrm{MeV}, \delta_{r, 1}=2.5, \delta_{r, 2}=3.5$, and $N_{\mathrm{r}, \text { tot }} \sim 10^{34}$ electrons distributed roughly uniformly over the loop length and isotropically over the pitch-angle. The thermal number density has almost no effect on this high-frequency emission: we varied the number density from $10^{10}$ to $5 \times 10^{11} \mathrm{~cm}^{-3}$ with essentially no modification of the spectrum. The sense of polarization corresponds to LCP wave at the high frequencies in agreement with observations, but the degree of polarization is much stronger than observed. The degree of polarization can be reduced by either a tangled magnetic field structure at the source or having a beam-like anisotropy of the accelerated electrons (Fleishman \& Melnikov 2003). We investigated possible effect of the beam-like anisotropy in our case and found that it offers a much better match (solid curves in Figure 10) to the measured degree of polarization at the impulsive peak than the isotropic distribution (dashed curves in Figure 10). The best fit is obtained for the number density of the fast electrons $n_{\mathrm{r}}=5.2 \times 10^{8} \mathrm{~cm}^{-3}$ (this is the peak value of the spatially nonuniform electron distribution) that corresponds to the total number of fast electrons at the source $N_{\text {r,tot }} \approx 1.35 \times 10^{34}$. Note, that the electron acceleration rate determined from the X-ray fit is about $1.2 \times 10^{35}$ electron $/ \mathrm{s}$, which implies that the electron escape time $\tau_{\text {esc }}$ from the loop is roughly $0.1 \mathrm{~s}$, which is three times larger than the time of flight ( $L_{\text {small }} / c \sim 30 \mathrm{~ms}$ ) estimated for our loop length $L_{\text {small }} \sim 9 \times 10^{8} \mathrm{~cm}$. Given the electron distribution is found to be beamed along the field lines, while the mirror ratio in this loop is small, $\sim 2$, a more reasonable estimate for the escape time would be within $30 \mathrm{~ms}$; this upper limit for the escape time is also implied by absence of any measurable (within $0.1 \mathrm{~s}$ time resolution) delay between the $35 \mathrm{GHz}$ light curve and HXR light curves. Our two-loop model offers a natural solution for this discrepancy: with the numbers above we conclude that in fact the acceleration rate is roughly two times larger than that derived from the HXR fit, but the remaining ( $~ 50 \%$ of) accelerated electrons escape to the second, big loop 14 rather than precipitate to the small loop footpoints; thus, they do not contribute to the HXR emission.

Apparently, we can estimate the escape time from the $35 \mathrm{GHz}$ light curve decay time $\tau$, which is roughly $1 \mathrm{~s}$, in even stronger contradiction with $0.03 \mathrm{~s}$ determined above. A reasonable way to reconcile this contradiction is to ascribe the decay segment of the light curve to the residual electron component trapped in the small loop. If so, this residual component must be substantially more isotropic than the original beamed distribution at the impulsive acceleration phase. The implied evolution of the pitch-angle distribution from a beamed to a more isotropic or loss-cone one must leave a fingerprint in the corresponding evolution of the degree of polarization. Indeed, as we noticed in

14 A fraction of the nonthermal electrons can also escape along the open field lines.

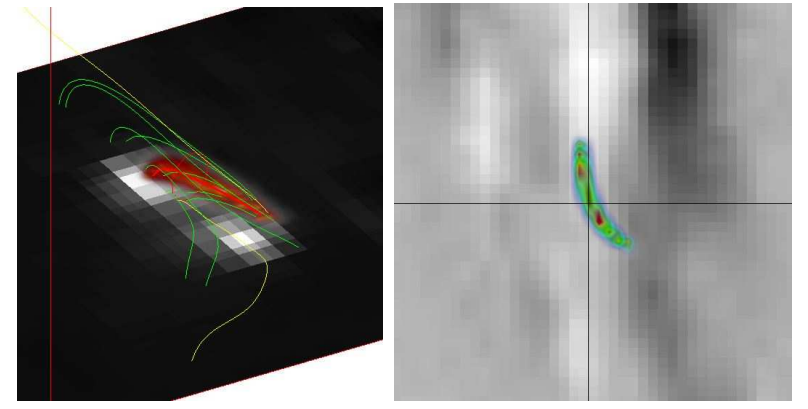

FIG. 9.- A small loop connecting two $195 \AA$ kernels built out of the LFFF extrapolation with $\alpha \approx-1.75 \times 10^{-9} \mathrm{~cm}^{-1}$. Left: perspective view with the EUV difference background image, magnetic structure visualized with a few closed (green) and open (yellow) field lines, cental field line of the flaring flux tube (red), and thermal electron density distribution (diffuse dark red volume). Right: number density of the fast electrons (diffuse green volume) on top of the LOS MDI magnetogram; top view.
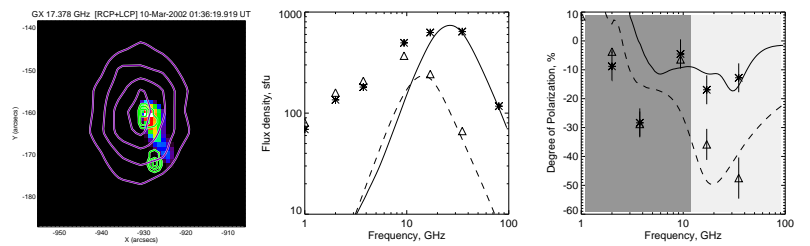

FIG. 10.- Key outcome of the small loop modeling of high-frequency emission (above $10 \mathrm{GHz}$ ). Left: synthetic microwave image at $17 \mathrm{GHz}$ computed from the adopted model along with EUV $195 \AA$ difference image (green contours) and NoRH $17 \mathrm{GHz}$ image of the south source (violet contours). Middle: observed total power spectrum at the microwave peak time (01:35:03.600 UT, asterisks) and early decay phase (01:35:08 UT, triangles) and the corresponding model spectra from a beam-like (solid line) and isotropic (dashed line) angular distributions. Right: observed degree of polarization at the same times and the corresponding polarization spectra for a beam-like (solid line) and isotropic (dashed line) angular distributions. Only the area on top of the light grey background is relevant for the model-to-data comparison.

Section 2.2, see Figure 2f, the degree of polarization goes up at 17 and $35 \mathrm{GHz}$ at the early decay phase. It is interesting that the polarization data at this decay phase are quantitatively consistent with the isotropic distribution of radiating electrons. Indeed, the model degree of polarization (dashed curve) agrees well with the data shown with the triangles in Figure 10, right. The model assumes the number density of the fast electrons $n_{\mathrm{r}}=6 \times 10^{7} \mathrm{~cm}^{-3}$ that corresponds to the total number of fast electrons at the source $N_{\mathrm{r}, \text { tot }} \approx 8 \times 10^{32}$, and the same spectral parameters as at the peak phase. The corresponding observed (triangles) and model (dashed curve) flux densities are shown in the middle panel of Figure 10.

Let us turn to the big loop modeling. We use now a bigger field of view covering both northern and southern microwave sources. Building a big loop in the right place is possible for extrapolated data cubes with positive $\alpha$ centered around the value $\alpha \approx 1.16 \times 10^{-9} \mathrm{~cm}^{-1}$. The center field line of the model loop has the length $L_{\mathrm{big}} \approx 8.2 \times 10^{9} \mathrm{~cm}$, the magnetic field value at the loop top ${ }^{15} B_{\mathrm{big}, \mathrm{lt}} \approx 30 \mathrm{G}$,

15 A scaling factor of 0.58 has been applied to the originally extrapolated magnetic data cube. 

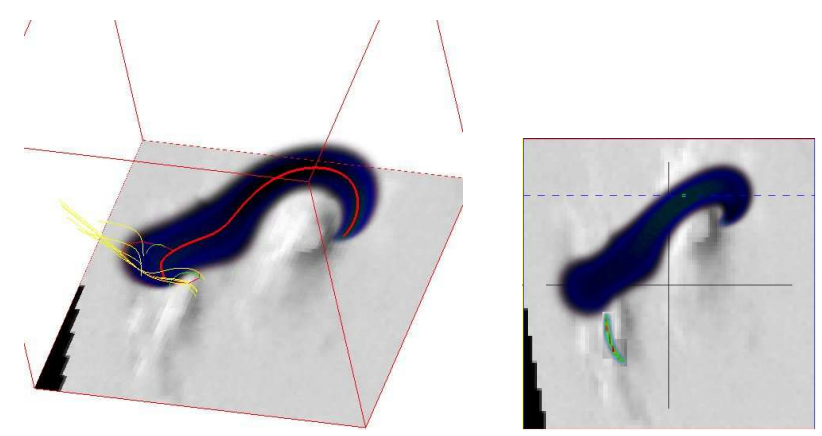

FIG. 11. - A big loop connecting two NoRH $17 \mathrm{GHz}$ sources (northern and southern) built out of the LFFF extrapolation with $\alpha \approx 1.16 \times 10^{-9} \mathrm{~cm}^{-1}$. Left: perspective view with the MDI LOS magnetogram background image, cental field line of the flaring flux tube is shown in red, and the nonthermal electron density distribution shown in diffuse dark volume. Right: top view with the small loop (green volume) added in scale.

and the mirror ratio about four, see Figure 11] We note that the nonthermal electron distribution in the big loop is poorer constrained than that in the small loop, because the accelerated electron distribution, before arriving at the big loop, is modified by the energy-dependent escape time from the small loop/acceleration region and then by the energydependent trapping time in the big loop. Moreover, we have only a limited information about the highfrequency slope of the microwave emission from the big loop from the spatially resolved NoRH data, while the total power NoRP data are dominated by the small loop as explained above. Given all these uncertainties, for the big loop we adopt a single powerlaw energy spectrum of the nonthermal electrons with the same (high-energy) spectral index as for the small loop, $\delta_{r}=3.5$.

For spectral modeling of the big loop contribution we select the time 01:35:24.500 UT at the decay phase - rather close to the end of the prominent spectral evolution, where emission from the big loop presumably dominates the microwave spectrum. We get a reasonably good spectral match at low frequencies if we populate this magnetic loop with fast electron distribution within the energy range starting from the same $E_{\min }=10 \mathrm{keV}$ in agreement with both HXR data and the small loop model to $E_{\max }=5 \mathrm{MeV} 16$ and the number density $n_{\mathrm{r}}=1.6 \times 10^{7} \mathrm{~cm}^{-3}$ totaling in $N_{\text {r,tot }} \approx 5.7 \times 10^{34}$ electrons slightly concentrating towards the looptop, as expected due to particle trapping effect in the magnetic loops (Melnikov et al. 2002). The angular distribution is expected to have a loss-cone shape with the loss-cone angle $\theta_{\mathrm{lt}}=30^{\circ}$ in the top of the loop in agreement with the mirror ratio of four. In fact, the isotropic distribution was found to give the same results, so we give here the numbers relevant to the isotropic model, which is computationally faster than the anisotropic one. The thermal plasma density at the central field line of the big loop is adopted to be $n_{0}=5 \times 10^{9} \mathrm{~cm}^{-3}$. This model offers a very good match for the low-frequency part of the

16 Although the value of $E_{\max }$ is poorly constrained by data, smaller $E_{\max }$ would result in a progressively stronger underestimation of the flux density at $34 \mathrm{GHz}$ from the northern source shown by open circle in Figure 12
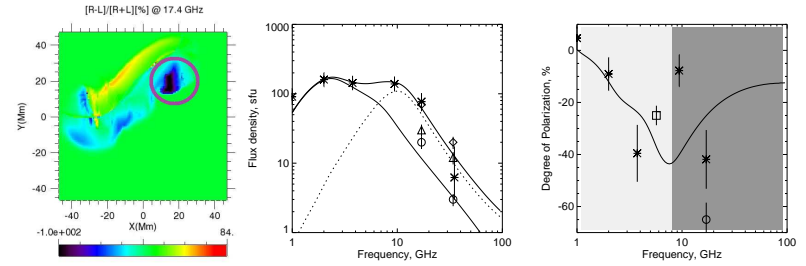

FIG. 12.- Key outcome of the big loop modeling. Left: synthetic microwave polarization image at $17 \mathrm{GHz}$ computed from the adopted model along with $\mathrm{NoRH} 17 \mathrm{GHz}$ highly polarized Northern source (violet contour). Middle: observed total power spectrum at the decay phase (01:35:24.500 UT; asterisks) and spatially resolved NoRH data at 17 and $34 \mathrm{GHz}$ from the northern source (open circles), the southern source (triangles), and total (diamonds) along with the corresponding model spectra from the big loop (thin solid line), the small loop (dotted line), and sum of them (thick solid line). Right: observed degree of polarization at the decay phase (01:35:24.500 UT) from NoRP (triangles), SSRT at $5.7 \mathrm{GHz}$ (square), and northern NoRH source at $17 \mathrm{GHz}$ (open circle) and the corresponding synthetic polarization spectrum produced by the big loop (solid line). Only the area on top of the light grey background is relevant for the model-to-data comparison.

total power spectrum and also reproduces the correct level of the spatially resolved data from the northern NoRH source at $17 \mathrm{GHz}$ and $34 \mathrm{GHz}$, although the flux density of the northern source at $17 \mathrm{GHz}$ is slightly underestimated. Similarly, the model slightly underestimates the flux density at $1 \mathrm{GHz}$, which indicates that the real source has a slightly stronger nonuniformity than the model one (cf. 3D models in Kuznetsov et al. 2011).

The model reproduces trends of the degree of polarization. Indeed, as it is seen from the right panel of Figure 12, the degree of polarization is close to zero at $1 \mathrm{GHz}$, while negative and relatively strong at $2 \mathrm{GHz}$ and $3.75 \mathrm{GHz}$ - all in agreement with observations. However, values of the model degree of polarization at $1 \mathrm{GHz}$ and $3.75 \mathrm{GHz}$ deviate from the observed values by a factor of two. We varied the angular distribution of the fast electrons in the big loop, but this did not remove the mismatch of the polarizations. We conclude that the mismatch is likely due to imperfect geometry of the model loop compared with the real source (recall, the degree of polarization is highly sensitive to the viewing angle), which is very possible given the limitations of our modeling due to lack of constraints discussed in the top of this section.

Finally, we checked and confirmed that the combination of these two loops is capable of matching the entire microwave spectrum at a given time; to illustrate that we selected the same time frame 01:35:24.500 UT, see the thick solid line in Figure12 Although the solution is far not unique, we can get a very good match to the entire spectrum between 1 and $35 \mathrm{GHz}$ by adding emission (dotted line in Figure 12) from a small loop with the same central field line as in the impulsive phase, but having a somewhat broader spatial distribution of the accelerated electrons transversely to this central field line. A consistent fast electron distribution has the number density of the fast electrons $n_{\mathrm{r}}=1.5 \times 10^{6} \mathrm{~cm}^{-3}$ that corresponds to the total number of fast electrons at the small loop $N_{\text {r,tot }} \approx 1.1 \times 10^{32}$ distributed isotropically with a modest concentration at the looptop 
(cf. Melnikov et al. 2002), and with the same broken power-law spectral shape. Given that we did not get the quantitative match of the degree of polarization at low frequencies, we did not try to match the entire polarization spectrum with the two-loop model.

With the described modeling we got a definitive 'yes' for questions 1-6 raised in the beginning of this section. The answer to the seventh question is quantitatively less definitive: we failed to quantitatively reproduce the degree of polarization at many frequencies or time frames analyzed. Nevertheless, we did obtain a generally right trend in all cases. Indeed, the sign of the degree of polarization is reproduced correctly for all time frames and at all frequencies; the model numbers are consistent with the observed ones within a factor of two. When we obtained a larger mismatch, we then found a much better match by adjusting the pitch-angle distribution of the fast electrons, which gave us additional important constraints on the model. We conclude that the proposed flare model based on two interacting loops is fully validated by the modeling performed here.

\section{DISCUSSION}

We have described a puzzling cold flare event observed in X-ray domain by GOES and Konus-Wind and in microwave domain by a number of instruments in a rather broad spectral range, covering more than two orders of magnitude in frequency - from $0.6 \mathrm{GHz}$ to $80 \mathrm{GHz}$. This flare displays a number of truly exceptional properties in all these domains. The HXR emission extends well above $1 \mathrm{MeV}$ and it displays one of the hardest energy spectrum ever detected (Vestrand et al. 1999) for a C-class flares. In SXR the GOES light curves are substantially delayed compared to what is expected according to the Neupert effect. In microwaves there is a number of peculiarities. The spectral peak frequency displays an exceptional variation over the burst between at least $35 \mathrm{GHz}$ at the impulsive peak down to $1.5 \mathrm{GHz}$ at the decay phase - the property observed for some of the strongest events, but clearly at odds for a modest C-class flare. The microwave light curves show a remarkable diversity of their shapes - from very impulsive and highly correlated with HXR light curves at $35 \mathrm{GHz}$ to more and more gradual at lower and lower frequencies - again, observed for a few X-class flares, but not for modest events. However, this exceptional spectral evolution takes place only over the impulsive phase and early decay phase, but then the spectral evolution switches off at some point and the microwave emission slowly decays with a constant spectral shape. In addition, during this remarkable spectral evolution stage, the coronal part of the microwave source moves rapidly eastward with the velocity of $320 \mathrm{~km} / \mathrm{s}$, but then stops and stays at the same location during the remaining decay phase.

The data analysis and 3D modeling suggest that all remarkable properties of this event can quantitatively be understood within a model involving energy release due to interaction of two non-potential magnetic flux tubes - one small and one big with different twists $\left(\alpha \approx-1.75 \times 10^{-9} \mathrm{~cm}^{-1}\right.$ and $\alpha \approx 1.16 \times$ $10^{-9} \mathrm{~cm}^{-1}$, respectively). Electrons are accelerated due to interaction (magnetic reconnection) between these two loops and then divided in roughly equal numbers between these two loops. The electrons injected into the small loop have a beam-like distribution directed towards the southern EUV kernel. This finding about the beamed pitch-angle distribution of the nonthermal electrons is highly important for understanding the electron acceleration and transport, yet no routine diagnostics of the electron angular distribution is available. Kontar \& Brown (2006) and Dickson \& Kontar (2013) used the effect of photospheric albedo on the HXR spectrum to conclude that nonthermal electron distribution is close to isotropic, while inconsistent with a noticeable beaming. However, this conclusion pertains to the chromospheric target volume, rather than to the coronal source. Fleishman (2006) employed microwave polarimetry to reveal a loss-cone anisotropy of trapped component of the nonthermal electrons, while Altyntsev et al. (2008, 2016) and Melnikov et al. (2014) reported the beam-like anisotropy for some events. It is interesting that Altyntsev et al. (2008, 2016) found the beam-like anisotropy in smaller loops in two events involving interaction between two different loops, which is in line with the finding discussed here.

Due to the beamed angular distribution most of the streaming nonthermal electrons immediately precipitate into the southern footpoint of the small loop and produce the HXR emission there. On the fly, they interact with the magnetic field of the loop, which is reasonably strong in the small loop, varying from $B \sim 600 \mathrm{G}$ at the looptop up to $B \sim 1200 \mathrm{G}$ at the footpoints, to produce the high-frequency microwave emission as observed. The total number of fast electrons, $N_{\text {r,tot }} \approx 1.35 \times 10^{34}$, needed to match the high-frequency part of the microwave spectrum at the peak time requires a roughly double acceleration rate as compared with that derived from the HXR thick-target model fit, $\sim 1.2 \times 10^{35}$ electron/s.

The missing electrons, those not seen via HXR emission, must have escaped to the big loop and be trapped there. To confirm this quantitatively, we note that at the decay phase time frame 01:35:24.500 UT, which we analyzed in great detail to validate the model, the total number of the trapped fast electrons was found to be $N_{\text {r,tot }} \approx 5.7 \times 10^{34}$ to match the microwave spectrum. This implies that at the peak time of the gradual microwave light curves (01:35:05 UT), when the flux density at $3.75 \mathrm{GHz}$ is twice bigger than at 01:35:24.500 UT, the number of the nonthermal electrons in the big loop must have been a factor of two larger, $N_{\text {r,tot }} \approx 1.2 \times 10^{35}$. This peak number of the fast electrons accumulated in the big loop is to be compared with the corresponding electron injection into the big loop. If we assume that the electron injection rate into the big loop is equivalent to the electron loss rate derived from the HXR thick-target spectral fit, the total number of electrons injected into the big loop would be $N_{\text {inj }} \sim 6 \times 10^{35}$ electrons over the impulsive phase of the flare; which, taken at the face value, is roughly five times larger than needed to supply the observed microwave emission from the big loop. Given that the number of the nonthermal electrons in the big loop is determined using a poorly 
defined low-energy spectral index and low-energy cutoff in the big loop, we conclude that the obtained electrons numbers are consistent with each other and so having a half of the accelerated electrons or slightly less to escape towards the big loop is sufficient to supply it with the required number of the fast electrons needed to match the low-frequency part of the microwave spectrum.

This picture is also quantitatively consistent with the light curves for various frequencies and energies. Indeed, the close correlation between the HXR (or $35 \mathrm{GHz}$ ) light curves and the time derivative of the $3.75 \mathrm{GHz}$ light curve is consistent with the former being a proxy of the acceleration/injection time profile, while the latter being a proxy of the trapped electron component. Now, the delay in the SXR GOES light curves becomes transparent: the direct losses of the accelerated electrons immediately available for plasma thermal response (including heating and evaporation) are limited to only a roughly half of all electrons, which precipitate through the small loop. The other half of the accelerated electrons trapped in the big loop lives longer and continues to heat the plasma via in situ Coulomb collisions in the loop and precipitation. This is why the GOES flux and the GOESderived temperature continue to grow well after the impulsive phase of the flare is over. We emphasize that such a scenario is only possible if the accelerated electrons are roughly equally divided between these two loops as is the case in our model, which additionally confirms the fast electron numbers obtained above for these two loops from the independent microwave spectrum fits.

Having the model validated, we can now address a number of fundamental questions about magnetic reconnection, particle acceleration, and transport. Recall, that the coronal microwave source moves quickly with the apparent velocity of roughly $320 \mathrm{~km} / \mathrm{s}$ passing about $35^{\prime \prime}$ over $80 \mathrm{~s}$ during the impulsive phase, which is reasonable to associate with a spread of the magnetic reconnection between the small and big loops. The process of magnetic reconnection will form new closed field lines (flux tubes), where the magnetic flux $\Phi$ is conserved along the field line (see, e.g., Qiu et al. 2009) such as $\Phi \sim V_{r} B_{r} \tau L_{r} \sim V_{\mathrm{lt}} B_{\mathrm{lt}} \tau L_{\mathrm{lt}}$, where $V_{r}, B_{r}$, and $L_{r}$ are the velocity, magnetic field, and spatial scale at the reconnection site (site of interaction between the loops), $V_{\mathrm{lt}}, B_{\mathrm{lt}}$, and $L_{\mathrm{lt}}$ are the same at the looptop, $\tau$ is the time of the reconnection process. Given that we know the magnetic field at the loop top from the modeling, $B_{\mathrm{big}, \mathrm{lt}} \approx 30 \mathrm{G}$, the looptop source velocity, and displacement, we can estimate the reconnecting electric field as $E_{r}[\mathrm{~V} / \mathrm{cm}] \sim$ $10^{-3} B_{\mathrm{lt}}[\mathrm{G}] V_{\mathrm{lt}}[\mathrm{km} / \mathrm{s}] \sim 10 \mathrm{~V} / \mathrm{cm}$. Since the magnetic field at the small loop is more than two orders of magnitude larger than $B_{\mathrm{lt}} \approx 30 \mathrm{G}$, the expected displacement of the reconnection site along the small loop is within $3^{\prime \prime}$ and unobservable in agreement with the constant location of the southern microwave source. Perhaps, this spatial extent of the reconnection is responsible for the increase of the small loop width required to get a good microwave fit at the decay phase.

This process of magnetic reconnection results, di- rectly or indirectly, in acceleration of a significant numbers of fast electrons to relativistic energies on a subsecond time scale. Interestingly, the microwave polarization data require that the accelerated electron distribution in the small loop is beamed along the magnetic field vector (i.e., from the northern source having the positive, north, magnetic polarity towards the southern source, having the negative, south, magnetic polarity). Recall, that the small loop has a negative $\alpha$, which implies that the electric field vector is directed oppositely to the magnetic field vector; thus, the fast electrons are beamed in the direction, where they are driven by the electric field; thus, the electric field is a likely cause of this electron beaming towards the southern footpoint.

The thermal electron number density in the small loop is poorly constrained; but given the fast electron number density is about $10^{9} \mathrm{~cm}^{-3}$, the total electron number density is at least that big. On the contrary, we can get a good estimate of the thermal electron number density at the big loop using the microwave spectral shape at the late decay phase. Indeed, the spectral peak frequency stays constant late in the event, $\approx 1.5 \mathrm{GHz}$, while the low-frequency spectral index is rather large, $\alpha_{\mathrm{lf}} \approx 3$, which are collectively indicative that the spectral peak is formed by Razineffect $f_{\text {peak }} \approx f_{R}$, rather than optical thickness effect, at the late decay phase. Given that the Razin frequency $f_{R} \approx 20 n_{0} / B$ and $B \approx 30 \mathrm{G}$, we estimate the mean thermal number density at the big loop as $n_{0} \sim(2-3) \times 10^{9} \mathrm{~cm}^{-3}$, which agrees well with the developed 3D model $\left(n_{0}=5 \times 10^{9} \mathrm{~cm}^{-3}\right.$ at the central field line of the loop and it decreases in the transverse direction over a gaussian law).

Note that the collisional loss time in such a tenuous plasma is longer that one minute for all electrons with energies higher than $100 \mathrm{keV}$ responsible for microwave emission, while the observed decay time of the light curve at $3.75 \mathrm{GHz}$, for example, is about $\tau \sim 30 \mathrm{~s}$. This unambiguously suggests that the highenergy fast electron loss from the big loop is mediated by the process of enhanced electron pitch-angle scattering by turbulence and their escape from the loop via the loss-cone. This is independently confirmed by lack of the electron spectral flattening, which must be present in case of collisionally-mediated electron transport.

In contrast, the low-energy electrons around the nominal low-energy cut-off of $10 \mathrm{keV}$ are likely strongly affected by in situ Coulomb losses. Indeed, the collisional loss time of $10 \mathrm{keV}$ electron in a plasma with number density around $2 \times 10^{9} \mathrm{~cm}^{-3}$ is about $3 \mathrm{~s}$. The corresponding energy deposition to the coronal plasma is roughly $\dot{E}=\int_{E_{\min }}^{E_{\max }} n_{r}(E) E / t_{E} d E \approx$ $0.2 \mathrm{erg} \mathrm{cm}^{-3} \mathrm{~s}^{-1}$, where $n_{r}(E)$ is the nonthermal electron distribution over energy and $t_{E}$ is the collisional loss time (cf. Sec. 4 in Bastian et al. 2007). Accordingly, the temperature increase over the interval $\tau$ is $\Delta T \sim \frac{\tau \dot{E}}{3 n_{0} k_{B}}$, where $k_{B}$ is the Boltzman constant; taking the observed duration of the main heating phase $\tau=30 \mathrm{~s}$ and plugging in other relevant 
numbers, we obtain $\Delta T \sim 7 \mathrm{MK}$ in agreement with GOES data. Thus, the energy-containing low-energy electrons deposit their energy directly to the coronal plasma, while the less numerous precipitating higherenergy electrons do not deposit sufficient energy to the footpoints to drive efficient chromospheric evaporation. This explains why we have a relatively strong microwave burst (which statistically corresponds to a M4-class event), but a rather weak C5 GOES flare. We conclude, that we have obtained a fully consistent picture of this cold flare event.

\section{CONCLUSIONS}

In this study we identified a new "cold" solar flare whose properties and physical model are substantially different from the cold flares reported so far (Bastian et al. 2007; Fleishman et al. 2011; Masuda et al. 2013). In contrast to the known cold flares, which consisted of one main loop, the described here 2002-03-10 cold flare is a vivid example of interaction between two loops. The first of them, a small one, is responsible for the impulsive flare component, while the bigger one is responsible for a more gradual nonthermal emission. Interestingly, the electrons accelerated in the event divided roughly evenly between these two loops, which made both loops comparably important in driving the thermal response in this event. For this reason the GOES flare was substantially delayed relative to the impulsive peak in apparent contradiction with the conventual Neupert effect. Deviations from the nominal Neupert effect have widely been reported (e.g., Veronig et al. 2002; Dennis et al. 2003; Veronig et al. 2005; Su et al. 2008) and often interpreted as an evidence in favor of an additional source of plasma heating. However, no additional heating is needed to understand the heating delay in our event: taking into account in situ coronal losses of the fast electron component trapped in the big loop, we obtained a scenario fully consistent with the plasma heating by the accelerated electrons - in a remarkable agreement with spirit of the Neupert effect. The developed model is in quantitative agreement with observations, including microwave imaging and polarization, and naturally identifies the cause of the suppressed chromospheric evaporation that is needed to interpret the unusually weak GOES response in this flare.

This work was supported in part by NSF grants AGS-1250374 and AGS-1262772, NASA grant NNX14AC87G to New Jersey Institute of Technology and RFBR grants 15-02-01089, 15-02-03717, 1502-03835, 15-02-08028, and 16-02-00749. This study was supported by the Program of basic research of the RAS Presidium No. 9. Authors acknowledge the Marie Curie PIRSES-GA-2011-295272 RadioSun project. We thank Dr. Gelu Nita for encouraging discussions.

\section{REFERENCES}

Altyntsev, A. A., Meshalkina, N. S., Fleishman, G. D., Myshyakov, I., \& Pal'shin, V. 2016, Sol. Phys., submitted

Altyntsev, A. T., Fleishman, G. D., Huang, G.-L., \& Melnikov, V. F. 2008, ApJ, 677, 1367

Aptekar, R. L., et al. 1995, Space Science Reviews, 71, 265 Arnaud, K. A. 1996, in Astronomical Society of the Pacific Conference Series, Vol. 101, Astronomical Data Analysis Software and Systems V, ed. G. H. Jacoby \& J. Barnes, 17

Aschwanden, M. J. 2005, Physics of the Solar Corona. An Introduction with Problems and Solutions (2nd edition) (Pour la Science)

Bastian, T. S., Benz, A. O., \& Gary, D. E. 1998, ARA\&A, 36, 131

Bastian, T. S., Fleishman, G. D., \& Gary, D. E. 2007, ApJ, 666,1256

Brown, J. C. 1971, Sol. Phys., 18, 489

Dennis, B. R. 1988, Sol. Phys., 118, 49

Dennis, B. R., Veronig, A., Schwartz, R. A., Sui, L., Tolbert, A. K., Zarro, D. M., \& Rhessi Team. 2003, Advances in Space Research, 32, 2459

Dickson, E. C. M., \& Kontar, E. P. 2013, Sol. Phys., 284, 405

Domingo, V., Fleck, B., \& Poland, A. I. 1995, Sol. Phys., 162, 1

Dulk. G. A. 1985, ARA\&A, 23, 169

Fleishman, G. D. 2006, in Solar Physics with the Nobeyama Radioheliograph, 51-62

Fleishman, G. D., Kontar, E. P., Nita, G. M., \& Gary, D. E. 2011, ApJ, 731, L19

-. 2013, ApJ, 768, 190

Fleishman, G. D., \& Kuznetsov, A. A. 2010, ApJ, 721, 1127

Fleishman, G. D., \& Melnikov, V. F. 2003, ApJ, 587, 823

Fleishman, G. D., Xu, Y., Nita, G. N., \& Gary, D. E. 2016, ApJ, 816, 62

Grechnev, V. V., et al. 2003, Sol. Phys., 216, 239

Guidice, D. A., Cliver, E. W., Barron, W. R., \& Kahler, S. 1981, in Bulletin of the American Astronomical Society,

Vol. 13, Bulletin of the American Astronomical Society, 553

Hanaoka, Y. 1997, Sol. Phys., 173, 319

Holman, G. D., et al. 2011, Space Sci. Rev., 159, 107

Kontar, E. P., \& Brown, J. C. 2006, ApJ, 653, L149

Kundu, M. R., Grechnev, V. V., White, S. M., Schmahl, E. J., Meshalkina, N. S., \& Kashapova, L. K. 2009, Sol. Phys., 260, 135
Kundu, M. R., White, S. M., Shibasaki, K., Sakurai, T., \& Grechnev, V. V. 2001, ApJ, 547, 1090

Kuznetsov, A. A., \& Kontar, E. P. 2015, Sol. Phys., 290, 79

Kuznetsov, A. A., Nita, G. M., \& Fleishman, G. D. 2011, ArXiv e-prints

Lee, J., \& Gary, D. E. 2000, ApJ, 543, 457

Lee, J., Gary, D. E., \& Shibasaki, K. 2000, ApJ, 531, 1109

Lee, J. W., Gary, D. E., \& Zirin, H. 1994, Sol. Phys., 152, 409

Lin, R. P., Dennis, B. R., Hurford, G. J., et al. 2002, Sol. Phys., 210, 3

Masuda, S., Shimojo, M., Kawate, T., Ishikawa, S.-n., \& Ohno, M. 2013, PASJ, 65, 1

Melnikov, V., Kuznetsov, S., \& Morgachev, A. 2014, in COSPAR Meeting, Vol. 40, 40th COSPAR Scientific Assembly

Melnikov, V. F. 1994, Radiophysics and Quantum Electronics, 37, 557

Melnikov, V. F., Gary, D. E., \& Nita, G. M. 2008, Sol. Phys., 253, 43

Melnikov, V. F., \& Magun, A. 1998, Sol. Phys., 178, 153

Melnikov, V. F., Shibasaki, K., \& Reznikova, V. E. 2002, ApJ, 580, L185

Nakajima, H., et al. 1994, IEEE Proceedings, 82, 705

Neupert, W. M. 1968, ApJ, 153, L59

Nita, G. M., Fleishman, G. D., Kuznetsov, A. A., Kontar, E. P., \& Gary, D. E. 2015, ApJ, 799, 236

Nita, G. M., Gary, D. E., \& Lee, J. 2004, ApJ, 605, 528

Pal'shin, V., et al. 2014, Geomagnetism and Aeronomy, 54, 943

Qiu, J., Gary, D. E., \& Fleishman, G. D. 2009, Sol. Phys., 255, 107

Schwartz, R. A., Csillaghy, A., Tolbert, A. K., Hurford, G. J., Mc Tiernan, J., \& Zarro, D. 2002, Sol. Phys., 210, 165

Somov, B. V., \& Syrovatskii, S. I. 1976, Soviet Physics Uspekhi, 19, 813

Stahli, M., Gary, D. E., \& Hurford, G. J. 1989, Sol. Phys., 120,351

Su, Y., Gan, W. Q., \& Li, Y. P. 2008, Advances in Space Research, 41, 988

Sui, L., Holman, G. D., \& Dennis, B. R. 2006, ApJ, 645, L157

—. 2007, ApJ, 670, 862 
Torii, C., Tsukiji, Y., S., K., Yoshimi, N., Tanaka, H., \& Enome, S. 1979, Proc. of the Res. Ist. of Atmospherics, Nagoya Univ., 26, 129

Veronig, A., Vršnak, B., Dennis, B. R., Temmer, M.

Hanslmeier, A., \& Magdalenić, J. 2002, A\&A, 392, 699

Veronig, A. M., Brown, J. C., Dennis, B. R., Schwartz, R. A.,

Sui, L., \& Tolbert, A. K. 2005, ApJ, 621, 482
Vestrand, W. T., Share, G. H., J. Murphy, R., Forrest, D. J., Rieger, E., Chupp, E. L., \& Kanbach, G. 1999, ApJS, 120, 409

\section{APPENDIX}

\section{THERMAL MODEL FOR THE GRADUAL FLARE COMPONENT}

Let us consider a model in which the thermal emission plays a role at low frequencies, while the nonthermal gyrosynchrotron emission dominates the high frequencies although it can give some contribution at the low frequencies. There are two main mechanisms of the thermal emission at the microwave range - free-free and gyro emission (gyroresonance, GR, or gyrosynchrotron, GS). Note that the opacity of the free-free emission decreases as the plasma temperature increases; thus, the plasma heating alone results in a decrease of the microwave free-free emission; its increase requires simultaneous significant increase of the plasma density to the numbers inconsistent with the emission measure $(E M)$ estimate available from the SXR GOES data; the peak value is $E M \approx 2 \times 10^{48} \mathrm{~cm}^{-3}$. Therefore, if the observed emission is thermal it can only be the gyro emission.

The weak polarization at $1 \& 2 \mathrm{GHz}$ tells us that the GR emission at these frequencies must be optically thick, while a significantly stronger polarization at $3.75 \& 5.7 \mathrm{GHz}$ implies that only the dominant X-mode (LCP in our case) may remain thick, while the O-mode (RCP) is getting thin here. As long as the thermal emission remains optically thick, its flux in each of the eigen-modes (X and $\mathrm{O}$ ) at a given frequency $f$ is firmly specified by a product of the plasma temperature $T$ and the source area $A$, such as

$$
F_{\mathrm{LCP}} \simeq F_{\mathrm{RCP}} \simeq 6[\mathrm{sfu}]\left(\frac{f}{1 \mathrm{GHz}}\right)^{2}\left(\frac{T}{10^{7} \mathrm{~K}}\right)\left(\frac{A}{10^{20} \mathrm{~cm}^{2}}\right),
$$

and the total flux is equal to the sum of the two components, $F=F_{\mathrm{LCP}}+F_{\mathrm{RCP}}$.

Let us consider first the implications of the thermal model for the emission at $5.7 \mathrm{GHz}$, where we have imaging data needed to estimate the source sizes and area. As has been estimated from Figure 7, left, the source sizes are $40^{\prime \prime} \times 90^{\prime \prime}$, i.e., $A \simeq 2 \times 10^{19} \mathrm{~cm}^{2}$, while $F_{\mathrm{LCP}} \simeq 100 \mathrm{sfu}$; thus, Eq. (A1) yields the plasma temperature around $T \sim 25 \mathrm{MK}$. This number looks somewhat excessive compared with the GOES-derived temperature, Figure 2d,e; however, it can still be fine if the plasma is tenuous and the corresponding $E M$ is small. Let us estimate the thermal plasma number density from the light curve cooling profile (the $5.7 \mathrm{GHz}$ light curve, not shown in Figure 2 is similar to that at $3.75 \mathrm{GHz}$ ). It is easy to estimate that the radiative cooling time is much longer than the observed decay time scale $\tau_{5.7 \mathrm{GHz}} \sim 30 \mathrm{~s}$; thus the cooling must be controlled by the thermal conduction, whose time scale is (cf. Eq. (4.3.10) in Aschwanden 2005)

$$
\tau \simeq 2.4 \cdot 10^{3}[\mathrm{~s}]\left(\frac{L}{10^{10} \mathrm{~cm}}\right)^{2}\left(\frac{n_{e}}{10^{10} \mathrm{~cm}^{-3}}\right)\left(\frac{10^{7} \mathrm{~K}}{T}\right)^{5 / 2}
$$

provided that the heat conduction has not yet reached the free-streaming limit. Therefore, to obtain the right time scale of the conductive cooling from the observed loop with the length $L \sim 10^{10} \mathrm{~cm}$ and temperature $T \sim 25$ MK requires $n_{e} \sim 10^{9} \mathrm{~cm}^{-3}$; being combined with the loop volume (assuming the sizes of $40^{\prime \prime} \times 40^{\prime \prime} \times 100^{\prime \prime}$ as observed) this density yields the emission measure $E M \sim 10^{46} \mathrm{~cm}^{-3}$, which is more than two orders of magnitude smaller than the GOES-derived background value, thus no GOES response is expected from this hot plasma. We conclude that the thermal model of the emission at $5.7 \mathrm{GHz}$ does not contradict available observational constraints.

Having said that, we have yet to check if this conclusion holds at lower frequencies of which we concentrate now on a lower one, $1 \mathrm{GHz}$. The problem is that radio flux from a uniform thermal source scales as $\propto f^{2}$ with frequency, see Eq. (A1), while we observe almost flat spectrum, which implies that the product $A \cdot T$ must scale roughly as $\propto f^{-2}$. We do not have spatially resolved measurements at $1 \mathrm{GHz}$, so let us first consider the case of the same temperature $T \sim 25$ MK but bigger area $A \sim 4 \cdot 10^{20} \mathrm{~cm}^{2}$ needed to provide the observed flux density at $1 \mathrm{GHz}$.

Given the implied increase of the source size at $1 \mathrm{GHz}$ compared with that at $5.7 \mathrm{GHz}$, the conductive cooling time is consistent with the observed value $\tau_{1 \mathrm{GHz}} \sim 150 \mathrm{~s}$ for roughly the same number density $n_{e} \sim 10^{9} \mathrm{~cm}^{-3}$. However, the enhanced source area also implies an enhanced volume, $V \sim 3 \cdot 10^{30} \mathrm{~cm}^{3}$, which yields the emission measure of $E M \sim 3 \cdot 10^{48} \mathrm{~cm}^{-3}$. Such an $E M$ would easily be revealed by GOES data on top of a comparable background value, which is not observed and, thus, calls the thermal model into question. On top of that, to get an optically thick thermal GR emission with the brightness temperature of $25 \mathrm{MK}$ from a given line of sight at $1 \mathrm{GHz}$, the line of sight must cross a volume element with $T \sim 25 \mathrm{MK}$ and the magnetic field equal to or exceeding $60 \mathrm{G}$, see Figure 13. Figure 7 right, shows the projected area of all lines of sight satisfying the condition $|B|>50 \mathrm{G}$ from the magnetogram directly, from which we can directly compute the maximum possible area of such a thermal gyro source as $A(>50 \mathrm{G}) \approx 4.77 \cdot 10^{19} \mathrm{~cm}^{2}$, which is by almost one order 


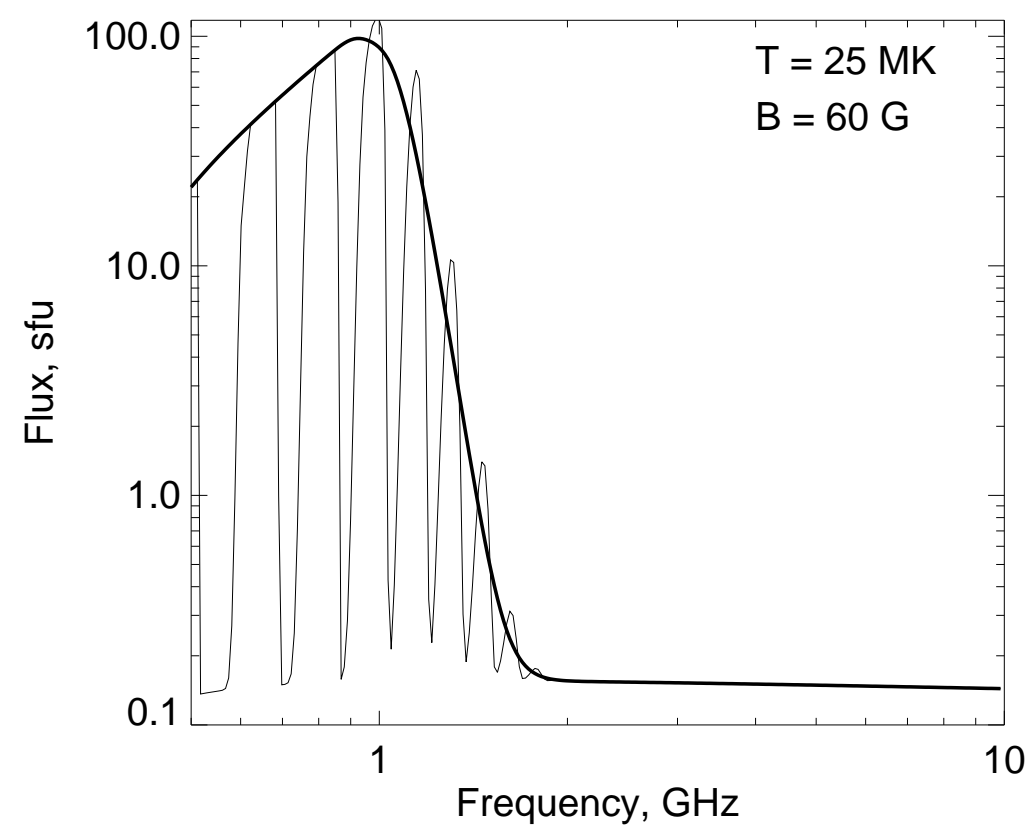

FIG. 13.- GS thermal spectrum plotted for a source with area $A=4 \times 10^{20} \mathrm{~cm}^{2}$, depth $d=7 \times 10^{8} \mathrm{~cm}$, T=25 MK, thermal density $n_{0}=10^{9} \mathrm{~cm}^{-3}, B=60 \mathrm{G}$, and the viewing angle $\theta=75^{\circ}$. Thin curve shows exact spectrum from a uniform source containing the gyroharmonics, while the thick curve shows the corresponding averaged spectrum obtained using the continuous fast code (Fleishman \& Kuznetsov 2010). Smaller magnetic field will result in lower spectral peak frequency.

of magnitude insufficient to reproduce the observed radio flux at $1 \mathrm{GHz}$. Our tests with 3D modeling using the extrapolated magnetic data cube confirm that even if we fill the entire data cube with the hot plasma having $T \sim 25 \mathrm{MK}$, the mismatch between the modeled and observed flux at $1 \mathrm{GHz}$ is more than a factor of two regardless of the selected combination of the input parameters. Having higher temperature would imply accordingly bigger density to keep the conductive cooling time the same as observed. But this enhanced density yields an enhanced EM in progressive disagreement with the GOES data. We conclude that the thermal model is not supported by the data. 